\title{
Competing Provers Protocols for Circuit Evaluation ${ }^{\dagger}$
}

\author{
Gillat Kol $\quad$ Ran $\mathrm{Raz}^{\S}$
}

April 21, 2014

\begin{abstract}
Let $C$ be a (fan-in 2) Boolean circuit of size $s$ and depth $d$, and let $x$ be an input for $C$. Assume that a verifier, that knows $C$ but doesn't know $x$, can access the low degree extension of $x$ at one random point. Two competing provers try to convince the verifier that $C(x)=0$ and $C(x)=1$, respectively, and assume that one of the provers is honest.

For any $r \geq 1$, we give an $r$ rounds protocol with communication complexity $d^{\frac{1}{r}}$ polylog $(s)$ that convinces the verifier of the correct value of $C(x)$ (with small probability of error). In particular, when we allow only one round, the protocol exchanges $d \cdot \operatorname{polylog}(s)$ bits, and when we allow $r=O\left(\frac{\log (d)}{\log \log (s)}\right)$ rounds, the protocol exchanges only polylog $(s)$ bits.

Moreover, the complexity of the verifier and honest prover in this protocol is poly $(s)$, and if in addition the circuit is $\log (s)$-space uniform, the complexity of the verifier is $d^{\frac{1}{r}} \operatorname{polylog}(s){ }^{1}$

The protocol is obtained by combining the delegation protocol of Goldwasser, Kalai and Rothblum [5] and the competing provers protocols of Feige and Kilian [3] and some new techniques. We suggest two applications of these results:
\end{abstract}

\footnotetext{
${ }^{\dagger}$ A conference version of this paper appeared in the Proceedings of the 4th Innovations in Theoretical Computer Science (ITCS), 2013 [8].

$\ddagger$ Research supported by Israel Science Foundation (ISF) grant.

${ }^{\S}$ Research supported by Israel Science Foundation (ISF) grant.

${ }^{1}$ We have learnt that a result similar to ours for the case $r=1$, as well as the motivation of delegating computation to competing clouds, was independently obtained by Canetti, Riva and Rothblum [2].
}

ACM Classification: H.4, F.0

AMS Classification: 68Q10, 68Q15

Key words and phrases: communication complexity, competing provers, delegation, complexity theory 
Delegating computation to competing clouds: The main motivation behind the protocol of [5] was delegating computation to a cloud. Using our new protocol, a verifier can delegate computation to two (or more) competing clouds. If at least one of the clouds is reliable the verifier can trust that the computation is correct (with high probability). The advantage over the protocol of [5] is that the communication complexity and the number of rounds in our protocol are significantly lower.

Communication complexity with competing provers, and circuit lower bounds: Aaronson and Wigderson [1] suggested the model of communication complexity with competing provers, where two competing provers try to convince two players that $f(x, y)=0$ and $f(x, y)=1$, respectively, where $x$ is an input held by the first player and $y$ is an input held by the second player. By scaling down the competing provers protocols of [3], they showed that strong enough lower bounds for the communication complexity of $f$, in this model, imply lower bounds for the computational complexity of $f$.

Our results strengthen this connection. More precisely, we show that if $f$ can be computed by a Boolean circuit of size $s$ and depth $d$ then for any $r \geq 1$ there is an $r$ rounds protocol for $f$, in this model, with communication complexity $d^{\frac{1}{r}}$ polylog $(s)$. This can be viewed as a possible direction towards proving circuit lower bounds. For instance, in order to prove $f \notin \mathrm{NC}$, it suffices to show that any one round protocol for $f$, in this model, requires the exchange of $\omega$ (polylog $(n))$ bits. This gives a relatively simple combinatorial property that implies strong circuit lower bounds.

\section{Introduction}

The model of refereed games, or, interactive proofs with competing provers, was first introduced by Feige, Shamir and Tennenholtz [4]. Informally, given a language $L$ and an input $x$, an efficient verifier interacts (that is, exchanges private messages) with two computationally-unbounded provers in order to decide whether or not $x \in L$. One prover tries to convince the verifier that $x \in L$, while the other prover tries to convince the verifier that $x \notin L$. (The assumption is that the prover who is right is honest and follows the protocol, while the other is untrusted). The seminal paper of Feige and Kilian [3] shows that the class of languages with a single round refereed games is exactly PSPACE, and the class of languages with $\operatorname{poly}(n)$ rounds refereed games is exactly EXP (the fact that EXP contains languages with poly $(n)$ rounds refereed games was shown by [9]).

Let $C$ be a (fan-in 2) Boolean circuit of size $s$ and depth $d$, and let $x$ be an input for $C$. Assume that a verifier that knows $C$ but doesn't know $x$ can access the low degree extension of $x$ (see Subsection 2.4 for a definition) at one random point. The verifier tries to decide whether or not $x$ satisfies $C$. Two competing provers try to convince the verifier that $C(x)=0$ and $C(x)=1$, respectively, and, as before, assume that one of the provers is honest.

Problems of this type in the model of interactive proofs with a single prover were studied in [6, 5]. In particular, the beautiful paper of Goldwasser, Kalai and Rothblum [5] gives a protocol where the prover convinces the verifier of the correct value of $C(x)$ (with a small probability of error), and where the number of rounds and the communication complexity are both $d \cdot \operatorname{polylog}(s)$, and the complexity of the prover is poly $(s)$. Moreover, maybe the most interesting aspect of their protocol is that if in addition 


\section{Competing Provers Protocols for Circuit Evaluation}

one assumes that the circuit $C$ is $\log (s)$-space uniform, the complexity of the verifier in the protocol is $d \cdot \operatorname{polylog}(s)$. That is, the complexity of the verifier may be significantly smaller than the size of the circuit. In other words, the computation was delegated to the prover, while the verifier is still convinced that the computation is correct.

\subsection{Our Results}

As before, let $C$ be a (fan-in 2) Boolean circuit of size $s$ and depth $d$, and let $x$ be an input for $C$. Assume that a verifier that knows $C$ but doesn't know $x$ can access the low degree extension of $x$ at one random point. Two competing provers try to convince the verifier that $C(x)=0$ and $C(x)=1$, respectively.

For any $r \geq 1$, we give an $r$ rounds protocol with communication complexity $d^{\frac{1}{r}} \operatorname{polylog}(s)$ that convinces the verifier of the correct value of $C(x)$ (with small probability of error). In particular, when we allow only one round, the protocol exchanges $d \cdot \operatorname{polylog}(s)$ bits, and when we allow $r=O\left(\frac{\log (d)}{\log \log (s)}\right)$ rounds, the protocol exchanges only polylog $(s)$ bits.

Moreover, the complexity of the verifier and (honest) provers in this protocol is $\operatorname{poly}(s)$, and if in addition the circuit is $\log (s)$-space uniform, the complexity of the verifier is $d^{\frac{1}{r}} \operatorname{polylog}(s)$.

The protocol is obtained by combining the delegation protocol of Goldwasser, Kalai and Rothblum [5] and the one round competing provers protocols for PSPACE of Feige and Kilian [3], together with some new techniques.

We have learnt that a result similar to ours for the case $r=1$, as well as the motivation of delegating computation to competing clouds, was independently obtained by Canetti, Riva and Rothblum [2].

\subsection{Delegating Computation to Competing Clouds}

As mentioned above, the main motivation behind the protocol of Goldwasser, Kalai and Rothblum [5] was delegating computation to a cloud: Consider the case of log-space uniform circuits. In particular, the class of languages decidable by log-space uniform circuits of polynomial size is exactly P. As mentioned above, the complexity of the verifier in this case is relatively small, assuming that she has access to the low degree extension of $x$ at a random point. Moreover, even if the verifier doesn't have access to the low degree extension of $x$ (but rather knows $x$ ), she can compute the low degree extension of $x$ at a random point by herself in almost linear time (that is, in time $n \cdot \operatorname{polylog}(n)$ ), which can be significantly smaller than the size of the circuit. Thus, the computation was delegated to the prover, while the verifier is convinced (with high probability) that the computation was performed correctly.

Using our new protocol, a verifier can delegate computation to two (or more) competing clouds, viewing each cloud as a separate prover. If at least one of the clouds is reliable, the verifier will be able to identify the correct answer and trust that the computation is correct (with high probability). The advantage over the protocol of [5] is that the communication complexity and the number of rounds in our protocol are significantly lower.

\subsection{Communication Complexity with Competing Provers and Circuit Lower Bounds}

Aaronson and Wigderson [1] suggested the model of communication complexity with two competing provers. 
Recall that the standard setting of communication complexity involves two separated, computationallyunbounded players, Alice and Bob, each holding a private $n$-bit input string. Assume that Alice holds $x \in\{0,1\}^{n}$ and Bob holds $y \in\{0,1\}^{n}$. Let $f:\{0,1\}^{2 n} \rightarrow\{0,1\}$ be a Boolean function known to both Alice and Bob, and let $x \circ y \in\{0,1\}^{2 n}$ denote the concatenation of $x$ and $y$. The players' mutual goal is to compute $f(x \circ y)$ with the least amount of communication between them.

In the model of communication complexity with competing provers the players may communicate, via private channels, with two provers that are assumed to know $f$, as well as both $x$ and $y$. The two provers try to convince the players that $f(x \circ y)=0$ and $f(x \circ y)=1$, respectively. The communication complexity of a protocol is the total number of bits exchanged between all the parties (players and provers). In this paper we are interested in both the number of rounds and the communication complexity of the suggested protocols.

By scaling down the competing provers protocols of [3], Aaronson and Wigderson showed that strong enough lower bounds for the communication complexity of $f$, in this model, imply lower bounds for the computational complexity of $f$. More precisely, they showed that for any language $L \in \mathrm{P}$ there are protocols in this model with poly-logarithmic rounds and communication complexity, and for any language $L \in$ LOGSPACE there are one-round protocols with poly-logarithmic communication complexity.

Our results strengthen this connection and relates the communication complexity of $f$ in this model to the circuit complexity of $f$.

More precisely, our results imply that if $f$ can be computed by a Boolean circuit of size $s$ and depth $d$ then for any $r \geq 1$ there is an $r$ rounds protocol for $f$, in this model, with communication complexity $d^{\frac{1}{r}}$ polylog $(s)$. In particular, as before, when we allow only one round, the protocol exchanges

$d \cdot \operatorname{polylog}(s)$ bits, and when we allow $r=O\left(\frac{\log (d)}{\log \log (s)}\right)$ rounds, the protocol exchanges only polylog $(s)$ bits.

This can be viewed as a possible direction towards proving circuit lower bounds. For instance, in order to prove $f \notin \mathrm{NC}$, it suffices to show that any one-round protocol for $f$, in this model, requires the exchange of $\omega$ (polylog $(n))$ bits. The one-round case is very appealing as it gives a relatively simple combinatorial condition that implies strong circuit lower bounds.

We also consider the model of communication complexity with one prover, where a single prover tries to convince the players that $f(x, y)=1$. We observe that the result of [5] (combined with the approach of [1]) implies a connection between the communication complexity of $f$ in this model and the circuit complexity of $f$. More precisely, their result implies that if $f$ can be computed by a Boolean circuit of size $s$ and depth $d$, then there are protocols for $f$, in this model, with $d$-polylog $(s)$ rounds and the exchange of $d \cdot \operatorname{polylog}(s)$ bits.

\section{Our Settings}

We study the query complexity and communication complexity settings in the presence of (untrusted) provers. In this section we present the formal settings used in the paper.

We start by reviewing the standard query and communication complexity settings (Subsection 2.1). We present a generalized framework for interaction between players and provers, called general communication protocols (Subsection 2.2). We describe the settings for query and communication complexity with 
provers (Subsections 2.3 and 2.4). Finally, we discuss the relations between query and communication complexity with provers (Subsection 2.5).

Let $\mathcal{F}_{n}$ be the set of Boolean functions on $n$ bits, $\mathcal{F}_{n}=\left\{f \mid f:\{0,1\}^{n} \rightarrow\{0,1\}\right\}$.

\subsection{Query and Communication Complexity}

\subsubsection{Query Complexity}

In the query complexity setting, we are given a function $f \in \mathcal{F}_{n}$ and an input $x \in\{0,1\}^{n}$. We are then asked to compute $f(x)$ by reading the least number of bits from $x$.

\subsubsection{Communication Complexity}

The communication complexity setting involves two separated, computationally-unbounded players, Alice and Bob, that attempt to evaluate a function $f \in \mathcal{F}_{2 n}$ known to both. Alice receives a private $n$-bit string $x$, and Bob receives a private $n$-bit string $y$. Let $x \circ y \in\{0,1\}^{2 n}$ denote the concatenation of the strings $x$ and $y$. The goal is for one of the players (for concreteness, say Alice) to compute and output $f(x \circ y)$, with the least amount of communication between them.

\subsection{General Communication Protocols}

We consider the setting in which a set of players and provers, each holding a private input (bit-string), communicate. A general communication protocol (GCP) prescribes a probabilistic strategy to each participant. During the execution of the protocol, any player may exchange messages (bit-strings) with any of the other players or provers through a private channel. We assume that there is no communication between the provers.

At the end of the protocol, the first player must output a value in $\{0,1, \perp\}$. Informally speaking, this value should be the result of the evaluation of a Boolean function on the players' inputs. A 0 or 1 output is interpreted as the actual value of the function, while $\perp$ indicates that one of the provers did not follow his prescribed strategy.

A GCP proceeds in rounds. Each round consists of three sets of messages sent in the following order (messages may be empty):

1. Players interact: Each player sends a message to each of the other players, according to some pre-specified order.

2. Players send questions: Each player sends a message (question) to each of the provers.

3. Provers reply: Each prover sends a message (answer) to each of the players.

The communication complexity of a GCP is defined as the maximal number of bits exchanged by all the involved parties (provers and players) in any execution. An $(r, c)$-GCP is a GCP with at most $r$ rounds, and communication complexity at most $c$. 


\subsection{Query and Communication Complexity with Provers}

Next, we formally define the settings used in the paper. We define two variants of the query complexity model, called QCP (Query Complexity with a Prover) and QCCP (Query Complexity with Competing Provers), as well as the two variants of the communication complexity model, called CCP (Communication Complexity with a Prover) and CCCP (Communication Complexity with Competing Provers).

In the CCP and CCCP models, the players attempt to compute the value of a function $f \in \mathcal{F}_{2 n}$ on an input $x \circ y \in\{0,1\}^{2 n}$. We assume that the prover(s) know both $x$ and $y$, while Alice has only $x$, and Bob has only $y$.

In the QCP and QCCP models, the player attempts to compute the value of a function $f \in \mathcal{F}_{n}$ on an input $x \in\{0,1\}^{n}$. We assume that the prover(s) have the input $x$, while the player has an oracle access to a (possibly a more "elaborate") version of $x$. That is, the player has access to a string $y=g(x)$, where $g$ is a function that possibly "stretches" $x$. We mention that in this paper $g(x)$ will always be the low-degree extension of $x$ (see Subsection 2.4).

In the QCP model, we require the player to output one of the values $f(x)$ or $\perp$ with probability at least $1-\varepsilon$, regardless of the behavior of the prover (that is, the player is only allowed to make a mistake with probability at most $\varepsilon$ ). If the prover is truthful (i.e., follows the prescribed strategy), we require the player to always output $f(x)$. The requirements for the CCP model are similar.

In the QCCP model, we assume that at least one of the provers is truthful (i.e., follows the prescribed strategy), and require the player to output $f(x)$ with probability at least $1-\varepsilon$, regardless of the behavior of the other prover. Note that, without loss of generality, we may assume that one prover tries to convince the player that $f(x)=1$, while the other tries to convince the player that $f(x)=0$. The requirements for the CCCP model are similar.

For the rest of the section let $\varepsilon \in[0,1], n, r, c, q, \ell \in \mathbb{N}, \mathbb{F}$ be a field, and $g:\{0,1\}^{n} \rightarrow \mathbb{F}^{\ell}$.

Definition 2.1 (QCP). QCP $\mathrm{Q}_{\varepsilon, g}(r, c, q)$ is the set of functions $f \in \mathcal{F}_{n}$ that can be computed by an $(r, c)$ GCP between a player and a prover, in the following sense: The prover is given an input $x \in\{0,1\}^{n}$, while the player is given the input $y=g(x)$.

For any strategy of the prover, a player who follows the protocol reads at most $q$ symbols from $y$ and, with probability at least $1-\varepsilon$, outputs either $f(x)$ or $\perp$. If the prover also follows the protocol, then the player always outputs $f(x)$.

Definition 2.2 (CCP). $\mathrm{CCP}_{\varepsilon}(r, c)$ is the set of functions $f \in \mathcal{F}_{2 n}$, that can be computed by an $(r, c)$ GCP between two players, Alice and Bob, and a prover, in the following sense: Alice is given an input $x \in\{0,1\}^{n}$ and Bob is given an input $y \in\{0,1\}^{n}$, while the prover is given the input $x \circ y$.

If both players follow the protocol, then for any strategy of the prover, with probability at least $1-\varepsilon$, Alice outputs either $f(x \circ y)$ or $\perp$. If the prover also follows the protocol, then Alice always outputs $f(x \circ y)$.

Definition 2.3 (QCCP). QCCP ${ }_{\varepsilon, g}(r, c, q)$ is the set of functions $f \in \mathcal{F}_{n}$, that can be computed by an $(r, c)$-GCP between a player and a pair of provers, in the following sense: Both provers are given (the same) input $x \in\{0,1\}^{n}$, while the player is given the input $y=g(x)$.

Assume that for any fixed $f$ and $x$, one of the provers, whose identity maybe unknown to the player, always follows the protocol. That is, this prover runs the probabilistic strategy prescribed to him by the 


\section{Competing Provers Protocols for Circuit Evaluation}

protocol. Then, for any strategy of the other prover, a player who follows the protocol reads at most $q$ symbols from $y$ and, with probability at least $1-\varepsilon$, outputs $f(x)$.

Definition $2.4(\mathrm{CCCP}) \cdot \mathrm{CCCP}_{\varepsilon}(r, c)$ is the set of functions $f \in \mathcal{F}_{2 n}$, that can be computed by an $(r, c)$ GCP between two players, Alice and Bob, and a pair of provers, in the following sense: Alice is given the input $x \in\{0,1\}^{n}$ and Bob is given the input $y \in\{0,1\}^{n}$, while both provers are given $x \circ y$.

Assume that for any fixed $f, x$ and $y$, one of the provers, whose identity maybe unknown to the players, always follows the protocol. That is, this prover runs the probabilistic strategy prescribed to him by the protocol. For any strategy of the other prover, if both players follow the protocol, Alice outputs $f(x \circ y)$ with probability at least $1-\varepsilon$.

\subsection{Low Degree Extension (LDE)}

Recall that in the QCP and QCCP models, the prover(s) have an input $x$, while the player has access to $y=g(x)$. As mentioned before, in this paper, $g(x)$ is always the low-degree extension of $x$. In this subsection we define the function $g(x)=\operatorname{LDE}(x)$, that maps $x$ to its low-degree extension.

Let $\mathbb{H}=\{0, \ldots, h-1\}$ be a set and $\mathbb{F}=\{0, \ldots, p-1\}$ be a field satisfying $h \leq p \in \mathbb{N}$. Recall that the Low-Degree Extension (LDE) of a function $x: \mathbb{H}^{m} \rightarrow \mathbb{F}$ is the unique $m$ variate polynomial $\tilde{x}: \mathbb{F}^{m} \rightarrow \mathbb{F}$ that agrees with $x$ on $\mathbb{H}^{m}$ (i.e., $\left.\tilde{x}\right|_{\mathbb{H}^{m}}=x$ ), and has degree at most $h-1$ in each of its $m$ variables.

We sometimes refer to $x$ and $\tilde{x}$ as truth table strings instead of functions. More precisely, we identify $x$ with the vector in $\mathbb{F}^{h^{m}}$ whose $j^{\text {th }}$ coordinate is $x\left(h_{j}\right)$, where $h_{j}$ is the $j^{\text {th }}$ element in the lexicographic ordering of $\mathbb{H}^{m}$. Similarly, we identify $\tilde{x}$ with the vector in $\mathbb{F}^{p^{m}}$ whose $j^{\text {th }}$ coordinate is $\tilde{x}\left(p_{j}\right)$, where $p_{j}$ is the $j^{\text {th }}$ element in the lexicographic ordering of $\mathbb{F}^{m}$.

Let $s \in \mathbb{N}$. We define a set $\mathbb{H}_{s}=\left\{0, \ldots, h_{s}-1\right\}$ and a field $\mathbb{F}_{s}=\left\{0, \ldots, p_{s}-1\right\}$ satisfying $h_{s} \leq p_{s} \in \mathbb{N}$. Let $h_{s}=\lceil\log (s)\rceil$. Let $m_{s} \in \mathbb{N}$ be the minimal such that $h_{s}^{m_{s}} \geq s$. Let $\mathbb{F}_{s}$ be the field of smallest size

satisfying $p_{s} \geq 12 m_{s} h_{s}^{3}$. Note that $h_{s}, p_{s} \leq$ polylog $(s), m_{s}=O\left(\frac{\log (s)}{\log \log (s)}\right)$ and $s \leq h_{s}^{m_{s}}, p_{s}^{m_{s}} \leq \operatorname{poly}(s)$.

For $n<s \in \mathbb{N}$, we define the function $\operatorname{LDE}_{s}:\{0,1\}^{n} \rightarrow \mathbb{F}_{s}^{p_{s}^{m_{s}}}$ as follows: Given $x \in\{0,1\}^{n}$, we pad $x$ with 1 's to get an $h_{s}^{m_{s}}$-size string $x^{\prime} \in \mathbb{F}_{s}^{h_{s}^{m_{s}}}$. That is, $x^{\prime}$ is defined by: $\forall i \in[n]: x_{i}^{\prime}=x_{i}$, and $\forall i \in\left\{n+1, \ldots, h_{s}^{m_{s}}\right\}: x_{i}^{\prime}=1$. We remark that we pad $x$ with 1's and not with 0's to make the proof of Lemma 5.1 (below) simpler. Finally, we define $\operatorname{LDE}_{s}(x)$ to be the low-degree extension $\tilde{x}^{\prime}$ of $x^{\prime}$.

\subsection{Connection Between Query and Communication Complexity with Provers}

The following observation made by [1] shows that results for the query complexity model (with $g(x)=$ $\mathrm{LDE}_{s}(x)$ ) imply analogous results for the communication complexity model. More precisely, in the communication complexity setting, any point in the low degree extension of the concatenated input $x \circ y$, can be computed by the players by the exchange of a single field element.

Let $S$ be a set, and let $c \in \mathbb{N}$. Consider the simple generalization of the standard communication complexity setting that allows Alice and Bob to exchange symbols from the set $S$ (instead of just bits). We denote by $\mathrm{CC}_{S}(c)$ the class of all functions $f:\{0,1\}^{2 n} \rightarrow S$ that can be computed by having Alice and Bob exchange at most $c$ elements of $S$.

Proposition 2.5 ([1]). Fix $n, s \in \mathbb{N}, s>2 n$, and $z \in \mathbb{F}_{s}^{m_{s}}$. Let $f:\{0,1\}^{2 n} \rightarrow \mathbb{F}_{s}$ be given by $f(x \circ y)=$ $\left(\operatorname{LDE}_{s}(x \circ y)\right)(z)$. Then, $f \in \mathrm{CC}_{\mathbb{F}_{s}}(1)$. 
The proof of Proposition 2.5 follows easily by the fact that low degree extension is a linear function.

We say that a QCP or a QCCP protocol is non-adaptive if the queries to $y=g(x)$ made by the player only depend on the player's random string. We mention that protocols presented in this paper are non-adaptive.

The following is an easy corollary of the above proposition.

Corollary 2.6. Let $f \in \mathcal{F}_{2 n}, r, c, q, s \in \mathbb{N}, 2 n<s$, and $\varepsilon \in[0,1]$.

1. Assume $f \in \mathrm{QCP}_{\varepsilon, \mathrm{LDE}_{s}}(r, c, q)$, with a non-adaptive protocol. Then $f \in \mathrm{CCP}_{\varepsilon}(r, c+O(q \cdot \log (s)))$.

2. Assume $f \in \mathrm{QCCP}_{\varepsilon, \mathrm{LDE}_{s}}(r, c, q)$, with a non-adaptive protocol. Then $f \in \mathrm{CCCP}_{\varepsilon}(r, c+O(q \cdot \log (s)))$.

Corollary 2.7. In the above CCP and CCCP protocols for $f$, the players exchange $c$ bits with the prover $(s)$, and $O(q \cdot \log (s))$ bits among themselves.

\section{Our Results}

Let $\mathcal{F}_{s, d}$ be the set of all functions $f \in \mathcal{F}_{2 n}$ that have an $s(n)$ size, $d(n)$ depth, Boolean circuit of fan-in 2 . The main result of this paper is that functions in $\mathcal{F}_{s, d}$ can be computed with low query and communication cost in the QCCP model, and therefore (due to Corollary 2.6) with low communication cost in the CCCP model.

The first theorem claims that in the QCP and CCP models, $d$ - polylog $(s)$ communication bits suffice in order to compute any $f \in \mathcal{F}_{s, d}$ with high probability. The QCP part of the theorem is a restatement of the main theorem of [5], while the CCP part of it follows as in Corollary 2.6.

Remark 3.1. In all the protocols below, the players and honest provers run in time at most poly $(s)$.

Theorem 3.2 ([5]). Let $n, s, d \in \mathbb{N}$ and $\varepsilon=\frac{1}{\log (s)}$. There exists a field $\mathbb{F}$ of size poly $(d, \log (s))$ and a function $^{1} g:\{0,1\}^{2 n} \rightarrow \mathbb{F}^{\text {poly(s) }}$, such that for every function $f \in \mathcal{F}_{s, d}$ it holds that

$$
f \in \mathrm{QCP}_{\varepsilon, g}(d \cdot \text { polylog }(s), d \cdot \text { polylog }(s), 1)
$$

and

$$
f \in \mathrm{CCP}_{\varepsilon}(d \cdot \text { polylog }(s), d \cdot \text { polylog }(s)) .
$$

Our next theorem shows that the computation of $f \in \mathcal{F}_{s, d}$ can be done with even lower communication complexity in the competing provers models. The theorem offers a trade-off between the number of rounds and the communication complexity of the protocol. Namely, for every $r \in \mathbb{N}$, there is a protocol that computes $f$ with high probability, and only requires $r$ rounds and $d^{\frac{1}{r}}$ polylog $(s)$ communication bits.

Theorem 3.3 (Main). Let $f \in \mathcal{F}_{s, d}$ and $\varepsilon=\frac{1}{\log (s)}$. For every $r \in \mathbb{N}, 1 \leq r \leq\lfloor\log (d)\rfloor$, it holds that

$$
f \in \mathrm{QCCP}_{\varepsilon, \mathrm{LDE}_{s}}\left(r, d^{\frac{1}{r}} \text { polylog }(s), 1\right)
$$

and

$$
f \in \operatorname{CCCP}_{\varepsilon}\left(r, d^{\frac{1}{r}} \text { polylog }(s)\right) .
$$

\footnotetext{
${ }^{1}$ The function $g$ is a low degree extension with different parameters than the ones we have here.
} 


\section{Competing Provers Protocols for Circuit Evaluation}

The next two theorems are easy corollaries of Theorem 3.3 that are obtained by taking $r$ to extreme values. Theorem 3.4 takes $r$ to be 1, and claims that $f$ can be computed by the exchange of $d$. polylog $(s)$ bits and with only a single communication round. Theorem 3.5 shows that by choosing $r=O\left(\frac{\log (d)}{\log \log (s)}\right)$ we can reduce the communication complexity of the protocol to polylog $(s)$ at the price of having $O\left(\frac{\log (d)}{\log \log (s)}\right)$ rounds.

Theorem 3.4. Let $f \in \mathcal{F}_{s, d}$ and $\varepsilon=\frac{1}{\log (s)}$. Then,

$$
f \in \mathrm{QCCP}_{\varepsilon, \mathrm{LDE}_{s}}(\mathbf{1}, d \cdot p \operatorname{polylog}(s), 1)
$$

and

$$
f \in \operatorname{CCCP}_{\varepsilon}(\mathbf{1}, d \cdot \operatorname{polylog}(s)) .
$$

Theorem 3.5. Let $f \in \mathcal{F}_{s, d}$ and $\varepsilon=\frac{1}{\log (s)}$. Then,

$$
\begin{gathered}
f \in \mathrm{QCCP}_{\varepsilon, \mathrm{LDE}_{s}}\left(O\left(\frac{\log (d)}{\log \log (s)}\right), \operatorname{polyl} \log (s), 1\right), \\
f \in \mathrm{CCCP}_{\varepsilon}\left(O\left(\frac{\log (d)}{\log \log (s)}\right), \text { polylog }(s)\right) .
\end{gathered}
$$

In order to prove Theorem 3.3, we first prove the special case for $r=1$ given by Theorem 3.4 (Section 6). We then show how to use additional rounds to reduce the communication complexity (Section 7).

The next theorem claims that if $f$ is given by a $\log (s)$-space uniform circuit of size $s$ and depth $d$, then the player(s) in Theorem 3.3 can be very efficient and run in time $d^{\frac{1}{r}}$ polylog $(s)$.

Theorem 3.6. Let $f \in \mathcal{F}_{s, d}$ with a $\log (s)$-space uniform circuit, and let $\varepsilon=\frac{1}{\log (s)} . \forall r \in \mathbb{N}$ the claim of Theorem 3.3 holds with players that run in time $d^{\frac{1}{r}}$ polylog $(s)$ (and honest provers that run in time poly $(s)$ ), and error $2 \varepsilon$.

Remark 3.7. Our protocols work with error $\varepsilon=\frac{1}{\log (s)}$. We mention that [2] offer an error reduction method for similar settings based on parallel repetition.

\section{Preliminaries}

Fix a size $s$ depth $d$ circuit $C$, and an input $x$ for $C$. Set $\varepsilon=\frac{1}{\log (s)}$. From now on, we omit the sub- $s$ and write $\mathbb{H}, \mathbb{F}, h, p, m$ instead of $\mathbb{H}_{s}, \mathbb{F}_{s}, h_{s}, p_{s}, m_{s}$. We enumerate the layers of $C$ such that layer 0 is the output layer and layer $d$ is the input layer. Note that since $s$ is the number of wires in $C$, the number of gates in each of the layers cannot exceed $s$.

We add gates to each of the layers of $C$, so that each will contain exactly $h^{m}>s$ gates. We label the gates in every layer by elements of $\mathbb{H}^{m}$. The gates added to every layer $i(i \in\{0, \ldots, d\})$, are all "special" gates that always return 1. The fact that every layer contains such a gate makes the proof of Lemma 5.1 (below) simpler. 
We associate with every layer $i \in\{0, \ldots, d\}$ the vector $V_{i} \in\{0,1\}^{h^{m}}$ (in particular, $V_{i}$ can be viewed as $V_{i} \in \mathbb{F}^{h^{m}}$ ) that consists of the values of the layer's gates when $C$ is evaluated on $x$. That is, for $j \in\left[h^{m}\right]$, the $j^{\text {th }}$ coordinate of $V_{i}$ is the value, when $C$ is evaluated on $x$, of the gate in layer $i$ whose label is the $j^{t h}$ element in the lexicographic ordering of $\mathbb{H}^{m}$. We denote by $\tilde{V}_{i} \in \mathbb{F}^{p^{m}}$ the low-degree extension of $V_{i}$.

We may assume, without loss of generality, that the output layer, layer 0 , originally consists of a single gate whose label is now $0^{m}$. The player's task can now be rephrased as computing $\tilde{V}_{0}\left(0^{m}\right)$.

Let $I=\{0, \ldots, d\}$ and $L=\{0, \ldots, 2 m\}$. Given a set $A$ and an element $a \in A$, we denote $A_{-a}=A \backslash\{a\}$. Let $\phi \in \mathbb{F}^{0}$ be the unique zero-coordinates vector. Whenever we say that a curve or a polynomial is of degree $k$, we mean that it is of degree at most $k$. A line is a degree 1 curve. All logarithms are taken with base 2 .

We will use the Schwartz-Zippel Lemma that bounds the probability that two different polynomials will agree at randomly selected test points.

Lemma 4.1 (Schwartz-Zippel Lemma). Let $\mathbb{F}$ be a field and let $P, Q \in \mathbb{F}\left[x_{1}, \ldots, x_{n}\right]$ be two different polynomials of total degree $d$. Let $S \subseteq \mathbb{F}$ be any finite subset. Then, by picking $y_{1}, \ldots, y_{n}$ independently and uniformly at random from $S$,

$$
\operatorname{Pr}\left[P\left(y_{1}, \ldots, y_{n}\right)=Q\left(y_{1}, \ldots, y_{n}\right)\right] \leq \frac{d}{|S|} .
$$

\section{$5 \quad$ Reducing Computation of Layer $i$ to Layer $i+1$}

In this section we follow the lines of [5] and show that the problem of computing $\tilde{V}_{i}$ on a point in $\mathbb{F}^{m}$ can be reduced to the task of computing $\tilde{V}_{i+1}$ on two points in $\mathbb{F}^{m}$, using a sum-check protocol.

\section{1 $\tilde{V}_{i}$ is a Quadratic Polynomial in the Values of $V_{i+1}$}

Let $i \in I_{-d}$. The next lemma claims that $\tilde{V}_{i}$ is a degree-2 polynomial in the values of $V_{i+1}$. The reason is that since $\tilde{V}_{i}$ is the low-degree extension of $V_{i}$, for every $z \in \mathbb{F}^{m}$, the value $\tilde{V}_{i}(z)$ is a linear combination of the values of $V_{i}$ on $\mathbb{H}^{m}$. That is, it is a linear combination of the values of the gates of layer $i$. In addition, the value of a gate in layer $i$ is a degree-2 polynomial in the values of its two children in layer $i+1$.

Lemma 5.1. For every $i \in I_{-d}$, there exists a function $\beta_{i}: \mathbb{F}^{m} \times\left(\mathbb{H}^{m}\right)^{2} \rightarrow \mathbb{F}$ that does not depend on the input $x$ to the circuit, such that:

1. $\forall z \in \mathbb{F}^{m}$ :

$$
\tilde{V}_{i}(z)=\sum_{w_{0}, w_{1} \in \mathbb{H}^{m}} \beta_{i}\left(z, w_{0}, w_{1}\right) V_{i+1}\left(w_{0}\right) V_{i+1}\left(w_{1}\right) .
$$

2. For every fixed $w_{0}, w_{1} \in \mathbb{H}^{m}$, and every $j \in[m]$, the function $\beta_{i}\left(z_{1} \ldots z_{m}, w_{0}, w_{1}\right)$ is a polynomial of degree at most $h-1$ in $z_{j}$.

Proof. Fix $i \in I_{-d}$ and let $z \in \mathbb{F}^{m}$. The value $\tilde{V}_{i}(z)$ can be written as

$$
\tilde{V}_{i}(z)=\sum_{p \in \mathbb{H}^{m}} \beta^{\prime}(z, p) \cdot V_{i}(p)
$$




\section{Competing Provers Protocols for Circuit Evaluation}

where $\beta^{\prime}(z, p): \mathbb{F}^{m} \times \mathbb{H}^{m} \rightarrow \mathbb{F}$ is the following $2 m$-variable function

$$
\beta^{\prime}(z, p)=\beta^{\prime}\left(z_{1} \ldots z_{m}, p_{1} \ldots p_{m}\right)=\prod_{j=1}^{m} \prod_{q_{j} \in \mathbb{H} \backslash\left\{p_{j}\right\}}\left(z_{j}-q_{j}\right) \cdot\left(p_{j}-q_{j}\right)^{-1} .
$$

Note that for every $j \in[m]$ and every fixed $p_{0} \in \mathbb{H}^{m}$, the function $\beta^{\prime}\left(z_{1} \ldots z_{m}, p_{0}\right)$ is a degree $h-1$ polynomial in $z_{j}$.

The value of gate $p \in \mathbb{H}^{m}$ in layer $i$ depends on the values of its two child gates in layer $i+1$. Recall that every layer of $C$ contains at least one "special" gate, that returns 1 on every input. Conclude that there exists a function $c:\left(\mathbb{H}^{m}\right)^{3} \rightarrow \mathbb{F}$ ( $c$ depends on the circuit $C$ ) such that

$$
V_{i}(p)=\sum_{w_{0}, w_{1} \in \mathbb{H}^{m}} c\left(p, w_{0}, w_{1}\right) V_{i+1}\left(w_{0}\right) V_{i+1}\left(w_{1}\right) .
$$

For example, if gate $p$ in layer $i$ is an OR gate whose children are gates $w_{0}, w_{1} \in \mathbb{H}^{m}$ in layer $i+1$, and gate $w^{*} \in \mathbb{H}^{m}$ in layer $i+1$ is a gate that always returns 1 , then

$$
\begin{gathered}
V_{i}(p)=V_{i+1}\left(w_{0}\right) V_{i+1}\left(w^{*}\right)+V_{i+1}\left(w_{1}\right) V_{i+1}\left(w^{*}\right) \\
-V_{i+1}\left(w_{0}\right) V_{i+1}\left(w_{1}\right) .
\end{gathered}
$$

Putting it all together,

$$
\begin{gathered}
\tilde{V}_{i}(z)= \\
\sum_{p \in \mathbb{H}^{m}} \beta^{\prime}(z, p) \cdot\left(\sum_{w_{0}, w_{1} \in \mathbb{H}^{m}} c\left(p, w_{0}, w_{1}\right) V_{i+1}\left(w_{0}\right) V_{i+1}\left(w_{1}\right)\right)= \\
\sum_{w_{0}, w_{1} \in \mathbb{H}^{m}}\left(\sum_{p \in \mathbb{H}^{m}} \beta^{\prime}(z, p) \cdot c\left(p, w_{0}, w_{1}\right)\right) V_{i+1}\left(w_{0}\right) V_{i+1}\left(w_{1}\right) .
\end{gathered}
$$

Finally, we denote

$$
\beta_{i}\left(z, w_{0}, w_{1}\right)=\sum_{p \in \mathbb{H}^{m}} \beta^{\prime}(z, p) \cdot c\left(p, w_{0}, w_{1}\right)
$$

and get

$$
\tilde{V}_{i}(z)=\sum_{w_{0}, w_{1} \in \mathbb{H}^{m}} \beta_{i}\left(z, w_{0}, w_{1}\right) V_{i+1}\left(w_{0}\right) V_{i+1}\left(w_{1}\right) .
$$

Since $\beta^{\prime}\left(z_{1} \ldots z_{m}, p\right)$ is of degree at most $h-1$ in $z_{j}$ for every $j \in[m]$, it is also the case that $\beta_{i}$ is of degree at most $h-1$ in $z_{j}$.

\subsection{Computing $\tilde{V}_{i}$ Given $\tilde{V}_{i+1}$ by Sum-Check}

Let $i \in I_{-d}$. The following lemma shows that for every $z \in \mathbb{F}^{m}$, verifying a claimed value for $\tilde{V}_{i}(z)$ can be done by a sum-check protocol that only requires the values of $\tilde{V}_{\mathbf{i}+\mathbf{1}}$ on two points in $\mathbb{F}^{m}$. 
Lemma 5.2. For every $i \in I_{-d}$, there exists a function $\tilde{\beta}_{i}:\left(\mathbb{F}^{m}\right)^{3} \rightarrow \mathbb{F}$ that does not depend on the input $x$ to the circuit, such that the function $\Phi_{i}:\left(\mathbb{F}^{m}\right)^{3} \rightarrow \mathbb{F}$ given by

$$
\Phi_{i}\left(z, w_{0}, w_{1}\right)=\tilde{\beta}_{i}\left(z, w_{0}, w_{1}\right) \tilde{V}_{i+1}\left(w_{0}\right) \tilde{V}_{i+1}\left(w_{1}\right)
$$

satisfies:

1. $\forall z \in \mathbb{F}^{m}: \tilde{V}_{i}(z)=\sum_{w_{0}, w_{1} \in \mathbb{H}^{m}} \Phi_{i}\left(z, w_{0}, w_{1}\right)$.

2. $\Phi_{i}$ is of degree at most $2(h-1)$ in each of its $3 m$ variables.

Proof. Fix $i \in I_{-d}$, let $z, w_{0}, w_{1} \in \mathbb{F}^{m}$, and let $\beta_{i}: \mathbb{F}^{m} \times\left(\mathbb{H}^{m}\right)^{2} \rightarrow \mathbb{F}$ be the function promised by Lemma 5.1. We denote by $\tilde{\beta}_{i}:\left(\mathbb{F}^{m}\right)^{3} \rightarrow \mathbb{F}$ the low-degree extension of $\beta_{i}\left(z, w_{0}, w_{1}\right)$ with respect to $w_{0}$ and $w_{1}$. That is, fix $z \in \mathbb{F}^{m}$, and let $\beta_{i, z}: \mathbb{H}^{2 m} \rightarrow \mathbb{F}$ be given by $\beta_{i, z}\left(w_{0}, w_{1}\right)=\beta_{i}\left(z, w_{0}, w_{1}\right)$. Let $\tilde{\beta}_{i, z}: \mathbb{F}^{2 m} \rightarrow \mathbb{F}$ be the low-degree extension of $\beta_{i, z}$. Finally, let $\tilde{\beta}_{i}\left(z, w_{0}, w_{1}\right)=\tilde{\beta}_{i, z}\left(w_{0}, w_{1}\right)$.

Due to Lemma 5.1, it holds that

$$
\forall z \in \mathbb{F}^{m}: \tilde{V}_{i}(z)=\sum_{w_{0}, w_{1} \in \mathbb{H}^{m}} \Phi_{i}\left(z, w_{0}, w_{1}\right) .
$$

Furthermore, $\Phi_{i}\left(z, w_{0}, w_{1}\right)$ is of low-degree in each of its $3 m$ variables: Let $z=z_{1} \ldots z_{m}, w_{0}=w_{0,1} \ldots w_{0, m}$, $w_{1}=w_{1,1} \ldots w_{1, m}$, and fix $j \in[m]$. Since $\tilde{\beta}_{i}$ is the low-degree extension of $\beta_{i}$ (with respect to $w_{0}$ and $w_{1}$ ), it is of degree at most $h-1$ in $w_{0, j}$ and $w_{1, j}$. Since $\tilde{V}_{i+1}$ is a low-degree extension of $V_{i+1}$, it is of degree at most $h-1$ in each of its $m$ variables. Conclude that $\Phi_{i}$ is of degree at most $2(h-1)$ in $w_{0, j}$ and $w_{1, j}$.

Fix $w_{0}, w_{1} \in \mathbb{H}^{m}$. Using Lemma 5.1, the function $\beta_{i}\left(z_{1} \ldots z_{m}, w_{0}, w_{1}\right)$ is a polynomial of degree $h-1$ in $z_{j}$. Again, using the fact that $\tilde{\beta}_{i}$ is the low-degree extension of $\beta_{i}$, it holds that $\tilde{\beta}_{i}\left(z, w_{0}, w_{1}\right)$ is a linear combination of the values $\beta_{i}\left(z, w_{0}^{\prime}, w_{1}^{\prime}\right)\left(w_{0}^{\prime}, w_{1}^{\prime} \in \mathbb{H}^{m}\right)$ with coefficients that depend only on $w_{0}, w_{1}, w_{0}^{\prime}, w_{1}^{\prime}$ (and are independent of $z$ ).

For every $w_{0}^{\prime}, w_{1}^{\prime} \in \mathbb{H}^{m}$, the value $\beta_{i}\left(z, w_{0}^{\prime}, w_{1}^{\prime}\right)$ is a polynomial of degree at most $h-1$ in $z_{j}$. Thus, any linear combination of these values, and in particular $\tilde{\beta}_{i}\left(z, w_{0}, w_{1}\right)$, is a polynomial of degree at most $h-1$ in $z_{j}$. Conclude that $\Phi_{i}$ is of degree at most $h-1$ in $z_{j}$.

For $i \in I_{-d}$, let $\Phi_{i}$ be the function defined in Lemma 5.2. For every $i \in I_{-d}, \ell \in L$, we define the partial-sum function $\Phi_{i, \ell}(z, x): \mathbb{F}^{m} \times \mathbb{F}^{\ell} \rightarrow \mathbb{F}$ as follows:

$$
\Phi_{i, \ell}\left(z, x_{1} \ldots x_{\ell}\right)=\sum_{x_{\ell+1}, \ldots, x_{2 m} \in \mathbb{H}} \Phi_{i}\left(z, x_{1} \ldots x_{2 m}\right) .
$$

Note that $\Phi_{i, \ell}\left(z_{1} \ldots z_{m}, x_{1} \ldots x_{\ell}\right)$ is of degree at most $2(h-1)$ in each of its $m+\ell$ variables, and that

$$
\begin{aligned}
\tilde{V}_{i}(z) & =\Phi_{i, 0}(z, \phi) \\
\Phi_{i}\left(z, w_{0}, w_{1}\right) & =\Phi_{i, 2 m}\left(z, w_{0} \circ w_{1}\right) .
\end{aligned}
$$

Also observe that a sum-check protocol can be used to reduce the computation of $\tilde{V}_{i}(z)=\Phi_{i, 0}(z, \phi)$ to the computation of $\Phi_{i}\left(z, w_{0}, w_{1}\right)=\Phi_{i, 2 m}\left(z, w_{0} \circ w_{1}\right)$. Since $\tilde{\beta}_{i}$ does not depend on the input to the circuit $x$, the player can compute $\Phi_{i, 2 m}\left(z, w_{0} \circ w_{1}\right)$ by herself given $\tilde{V}_{i+1}\left(w_{0}\right)$ and $\tilde{V}_{i+1}\left(w_{1}\right)$. In other words, the evaluation of $\tilde{V}_{i}$ on $z$ can be reduced, by a sum-check protocol, to the computation of $\tilde{V}_{i+1}$ on the pair of points $w_{0}, w_{1} \in \mathbb{F}^{m}$. 


\section{One Round Protocol}

In this section we prove Theorem 3.4. Namely, we show that for any $f \in \mathcal{F}_{s, d}$ it holds that $f \in$ $\operatorname{QCCP}_{\varepsilon, \operatorname{LDE}_{s}}(1, d \cdot \operatorname{polylog}(s), 1)$ where $\varepsilon=\frac{1}{\log (s)}$. We describe a protocol that given a size $s$ depth $d$ circuit $C$ on $n$ bits, and an input $x \in\{0,1\}^{n}$, outputs $C(x)$ with probability at least $1-\varepsilon$. The protocol consists of a single round, requires the exchange of $d \cdot \operatorname{polylog}(s)$ bits, and makes a single query to $\tilde{x}=\operatorname{LDE}_{s}(x)$.

The protocol consists of three steps: (i) The player sends questions to the provers (Subsection 6.1). (ii) The provers reply (Subsection 6.2). (iii) Using the provers' answers, the player decides on the value of $C(x)$ (Subsection 6.4). Subsection 6.3 contains observations that are used in Subsection 6.4 in order to show the correctness of the player's decision.

We assume that one of the provers claims that $C(x)=1$, while the other claims $C(x)=0$. Otherwise, if both claim that $C(x)=b(b \in\{0,1\})$, then since at least one of the provers is truthful, it must be the case that indeed $C(x)=b$. In the protocol below we assume that the player knows the identity of the prover claiming that $C(x)=1$ (we call him $\mathrm{P}_{1}$ ), and the identity of the prover claiming that $C(x)=0$ (we call him $\mathrm{P}_{0}$ ). This assumption is justified as the player may require each prover to add his claimed value for $C(x)$ to his reply message. (The proof for the case that $\mathrm{P}_{1}$ claims $C(x)=0$ and $\mathrm{P}_{0}$ claims $C(x)=1$ is similar).

For the rest of the section let $\beta \in \mathbb{F} \backslash \mathbb{H}$ be an arbitrary element.

Remark 6.1 (functions representation). During the execution of the protocol, the participants exchange a set of curves and polynomials. Each of the exchanged curves is of degree (at most) $h$. Each exchanged polynomial is either of the form $F(r)=\tilde{V}_{i}(Z(r))(i \in I)$ or of the form $F(r, t)=\Phi_{i, \ell}(Z(r), X(t))$ $\left(i \in I_{-d}, \ell \in L\right)$, where $Z(r): \mathbb{F} \rightarrow \mathbb{F}^{m}$ and $X(t): \mathbb{F} \rightarrow \mathbb{F}^{\ell}$ are two previously exchanged degree- $h$ curves. In other words, the exchanged polynomials are all restrictions of $\tilde{V}_{i}$ and $\Phi_{i, \ell}$ to degree $h$ curves.

The polynomial $\tilde{V}_{i}(z)$ is of degree at most $h-1$ in each of its $m$ variables, as it is the low-degree extension of $V_{i}$. The polynomial $\Phi_{i, \ell}\left(z, x_{1} \ldots x_{\ell}\right)$ is of degree at most $2(h-1)$ in each of its $m+\ell$ variables (see Subsection 5.2). Therefore, each exchanged polynomial is of total degree at most $h$. $2(h-1) \cdot(m+\ell)<6 m h^{2}$. We require each such polynomial to be sent in a canonical representation, as a sequence of at most $6 \mathrm{mh}^{2}$ coefficients. Curves will be sent as sequences of polynomials.

\subsection{The Player's Questions}

For every $i \in I_{-d}, \ell \in L$, the player constructs a degree- $h$ curve $C_{i, \ell}: \mathbb{F} \rightarrow \mathbb{F}^{\ell}$, and a line $D_{i, \ell}: \mathbb{F} \rightarrow \mathbb{F}^{\ell}$. For every $i \in I$, the player constructs a degree-2 curve $Z_{i}: \mathbb{F} \rightarrow \mathbb{F}^{m}$, and a point $z_{i} \in \mathbb{F}^{m}$. The curves $Z_{i}(i \in I)$ and $C_{i, \ell}\left(i \in I_{-d}, \ell \in L\right)$ are given to $\mathrm{P}_{1}$, while the points $z_{i}(i \in I)$ and the lines $D_{i, \ell}\left(i \in I_{-d}, \ell \in L\right)$ are given to $\mathrm{P}_{0}$.

Let $Z_{0}: \mathbb{F} \rightarrow \mathbb{F}^{m}$ be the constant $0^{m}$ curve. That is, $\forall r \in \mathbb{F}: Z_{0}(r)=0^{m}$. Randomly select $\zeta_{0} \in_{R}$ $\mathbb{F} \backslash\{0,1\}$. Set $z_{0}=Z_{0}\left(\zeta_{0}\right)=0^{m}$.

Recall that $\beta \in \mathbb{F} \backslash \mathbb{H}$ is an arbitrary element. For $i=0, \ldots, d-1$ we construct the additional needed curves and points in the following order:

The curves $\left(C_{i, \ell}\right)_{\ell \in L}$ and $\left(D_{i, \ell}\right)_{\ell \in L}$ are constructed recursively: 
- Construct $C_{i, 0}, D_{i, 0}:$ Define $C_{i, 0}, D_{i, 0}: \mathbb{F} \rightarrow \mathbb{F}^{0}$ by $\forall t \in \mathbb{F}: C_{i, 0}(t)=D_{i, 0}(t)=\phi$.

- Construct $C_{i, \ell}$ given $D_{i, \ell-1}\left(\ell \in L_{-0}\right)$ :

Randomly select $\delta_{i, \ell-1} \in_{R} \mathbb{F} \backslash\{0\}$ and set $a_{i, \ell-1}=D_{i, \ell-1}\left(\delta_{i, \ell-1}\right)$.

Let $C_{i, \ell}$ be a random degree- $h$ curve satisfying $\forall \alpha \in \mathbb{H}: C_{i, \ell}(\alpha)=a_{i, \ell-1} \circ \alpha$, chosen as follows: Randomly select $c_{i, \ell} \in R \mathbb{F}^{\ell}$, and let $C_{i, \ell}$ be the degree- $h$ curve satisfying $\forall \alpha \in \mathbb{H}: C_{i, \ell}(\alpha)=$ $a_{i, \ell-1} \circ \alpha$ and $C_{i, \ell}(\beta)=c_{i, \ell}$.

- Construct $D_{i, \ell}$ given $C_{i, \ell}\left(\ell \in L_{-0}\right)$ :

Randomly select $\gamma_{i, \ell} \in_{R} \mathbb{F} \backslash \mathbb{H}$.

Let $D_{i, \ell}$ be a random line satisfying $D_{i, \ell}(0)=C_{i, \ell}\left(\gamma_{i, \ell}\right)$, chosen as follows: Randomly select $d_{i, \ell} \in_{R} \mathbb{F}^{\ell}$, and let $D_{i, \ell}$ be the line satisfying $D_{i, \ell}(0)=C_{i, \ell}\left(\gamma_{i, \ell}\right)$ and $D_{i, \ell}(1)=d_{i, \ell}$.

The curve $Z_{i+1}$ is constructed as follows: Randomly select $\delta_{i, 2 m} \in_{R} \mathbb{F} \backslash\{0\}$ and set $a_{i, 2 m}=D_{i, 2 m}\left(\delta_{i, 2 m}\right)$. Denote $a_{i, 2 m}=w_{i, 0} \circ w_{i, 1}$ where $w_{i, 0}, w_{i, 1} \in \mathbb{F}^{m}$.

Let $Z_{i+1}$ be a random degree-2 curve satisfying $Z_{i+1}(0)=w_{i, 0}$ and $Z_{i+1}(1)=w_{i, 1}$, chosen as follows: Randomly select $w_{i+1} \in \mathbb{F}^{m}$, and let $Z_{i+1}$ be the degree- 2 curve satisfying $Z_{i+1}(0)=w_{i, 0}, Z_{i+1}(1)=w_{i, 1}$ and $Z_{i+1}(2)=w_{i+1}$.

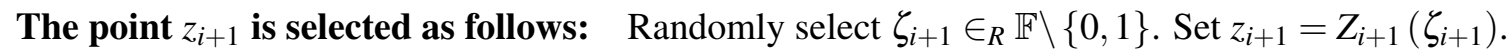

\subsection{The Provers' Answers}

\section{$P_{1}$ 's reply}

For $i \in I_{-d}, \ell \in L_{-0}$, denote by $T_{i, \ell}^{*}: \mathbb{F} \times \mathbb{F} \rightarrow \mathbb{F}$ the polynomial $T_{i, \ell}^{*}(r, t)=\Phi_{i, \ell}\left(Z_{i}(r), C_{i, \ell}(t)\right)$. For $i \in I_{-0}$, denote by $U_{i}^{*}: \mathbb{F} \rightarrow \mathbb{F}$ the polynomial $U_{i}^{*}(r)=\tilde{V}_{i}\left(Z_{i}(r)\right)$.

$\mathrm{P}_{1}$ replies with a set of polynomials $T_{i, \ell}: \mathbb{F} \times \mathbb{F} \rightarrow \mathbb{F}\left(i \in I_{-d}, \ell \in L_{-0}\right)$, and a set of polynomials $U_{i}: \mathbb{F} \rightarrow \mathbb{F}\left(i \in I_{-0}\right)$. The honest $\mathrm{P}_{1}$ should reply with $T_{i, \ell}=T_{i, \ell}^{*}$ and $U_{i}=U_{i}^{*}$.

\section{$\mathrm{P}_{0}$ 's reply}

For $i \in I_{-d}, \ell \in L$, denote by $Q_{i, \ell}^{*}: \mathbb{F} \rightarrow \mathbb{F}$ the polynomial $Q_{i, \ell}^{*}(t)=\Phi_{i, \ell}\left(z_{i}, D_{i, \ell}(t)\right)$.

$\mathrm{P}_{0}$ replies with a set of polynomials $Q_{i, \ell}: \mathbb{F} \rightarrow \mathbb{F}\left(i \in I_{-d}, \ell \in L\right)$. The honest $\mathrm{P}_{0}$ should reply with $Q_{i, \ell}=Q_{i, \ell}^{*}$

\subsection{Propositions}

This subsection contains propositions that will be useful in the analysis of the player's decision in Subsection 6.4. Propositions 1 and 2 claim that the information given to $P_{1}$ by the player is independent of the pairs of values $\left(\zeta_{i}, \gamma_{i, \ell}\right)$. Propositions 3 and 4 claim that the information given to $\mathrm{P}_{0}$ by the player is independent of the values $\delta_{i, \ell}$. 


\section{Competing Provers Protocols for Circuit Evaluation}

Denote by $\operatorname{In} f_{1}$ and $\operatorname{In} f_{0}$ the random variables describing the information given to $\mathrm{P}_{1}$ and $\mathrm{P}_{0}$ (respectively):

and

$$
\operatorname{Inf}_{1}=\left(\left(Z_{i}\right)_{i \in I},\left(C_{i, \ell}\right)_{i \in I_{-d}, \ell \in L}\right)
$$

$$
\operatorname{Inf} f_{0}=\left(\left(z_{i}\right)_{i \in I},\left(D_{i, \ell}\right)_{i \in I_{-d}, \ell \in L}\right) .
$$

In the proofs below, whenever we say "object" we mean a field element, point or curve selected by the player while constructing the questions to the provers.

Proposition 1. Let $i \in I_{-d}, \ell \in L_{-0} . \gamma_{i, \ell}$ is independent of $\operatorname{In} f_{1}$. That is, $\gamma_{i, \ell}$ is uniformly distributed in $\mathbb{F} \backslash \mathbb{H}$, even given $\operatorname{Inf} f_{1}$.

Proof. Let $X$ be a random variable consisting of all the curves $Z_{i^{\prime}}$ and $C_{i^{\prime}, \ell^{\prime}}$ selected by the player before $\gamma_{i, \ell}$ is selected. Let $Y$ be a random variable consisting of all the curves $Z_{i^{\prime}}$ and $C_{i^{\prime}, \ell^{\prime}}$ selected after $\gamma_{i, \ell}$. Clearly, $\gamma_{i, \ell}$ is selected independently of $X$. We will show that $Y$ is independent of the pair $\left(X, \gamma_{i, \ell}\right)$. This implies that $\operatorname{In} f_{1}$ is independent of $\gamma_{i, \ell}$.

The line $D_{i, \ell}$ was selected to be a random line satisfying $D_{i, \ell}(0)=C_{i, \ell}\left(\gamma_{i, \ell}\right)$. Therefore, for every fixed $\delta \in \mathbb{F} \backslash\{0\}$, the value $D_{i, \ell}(\delta)$ is uniformly distributed in $\mathbb{F}^{\ell}$, even given all the objects selected before $D_{i, \ell}$. In particular, $a_{i, \ell}=D_{i, \ell}\left(\delta_{i, \ell}\right)$ is uniformly distributed in $\mathbb{F}^{\ell}$, even given all objects selected before $D_{i, \ell}$.

The player can select all the objects selected after $D_{i, \ell}$ by only knowing $a_{i, \ell}$. Since $a_{i, \ell}$ is uniformly distributed in $\mathbb{F}^{\ell}$, even given all objects selected before $D_{i, \ell}$, it holds that the objects selected after $D_{i, \ell}$ are independent of all objects selected before $D_{i, \ell}$. In particular, $Y$ is independent of the pair $\left(X, \gamma_{i, \ell}\right)$.

Proposition 2. Let $i \in I_{-d}, \ell \in L_{-0} . \zeta_{i}$ is independent of the pair $\left(\operatorname{Inf} f_{1}, \gamma_{i, \ell}\right)$. That is, $\zeta_{i}$ is uniformly distributed in $\mathbb{F} \backslash\{0,1\}$, even given $\left(\operatorname{In} f_{1}, \gamma_{i, \ell}\right)$. Furthermore, $\zeta_{d}$ is independent of $\operatorname{In} f_{1}$.

Proof. Clearly, $\zeta_{i}$ is uniformly distributed, even given all the previously selected objects. In addition, all the objects selected after $\zeta_{i}$ (and after $z_{i}$ was set) are independent of all the objects selected up to $\zeta_{i}$ (including $\zeta_{i}$ ).

Proposition 3. Let $i \in I_{-d}, \ell \in L_{-2 m} . \delta_{i, \ell}$ is independent of $I n f_{0}$. That is, $\delta_{i, \ell}$ is uniformly distributed in $\mathbb{F} \backslash\{0\}$, even given $\operatorname{Inf} f_{0}$.

Proof. Let $X$ be a random variable consisting of all the points $z_{i^{\prime}}$ and curves $D_{i^{\prime}, \ell^{\prime}}$ selected by the player before $\delta_{i, \ell}$ is selected. Let $Y$ be a random variable consisting of all the points $z_{i^{\prime}}$ and curves $D_{i^{\prime}, \ell^{\prime}}$ selected after $\delta_{i, \ell}$. Clearly, $\delta_{i, \ell}$ is selected independently of $X$. We will show that $Y$ is independent of the pair $\left(X, \delta_{i, \ell}\right)$. This implies that $\operatorname{In} f_{0}$ is independent of $\delta_{i, \ell}$.

The curve $C_{i, \ell+1}$ was selected to be a random degree- $h$ curve satisfying $\forall \alpha \in \mathbb{H}: C_{i, \ell+1}(\alpha)=a_{i, \ell} \circ \alpha$. Therefore, for every fixed $\gamma \in \mathbb{F} \backslash \mathbb{H}$, the value $C_{i, \ell+1}(\gamma)$ is uniformly distributed in $\mathbb{F}^{\ell+1}$, even given all objects selected before $C_{i, \ell+1}$. In particular, $C_{i, \ell+1}\left(\gamma_{i, \ell+1}\right)$ is uniformly distributed in $\mathbb{F}^{\ell+1}$, even given all objects selected before $C_{i, \ell+1}$.

The player can select all the objects selected after $C_{i, \ell+1}$ by only knowing $C_{i, \ell+1}\left(\gamma_{i, \ell+1}\right)$. Since $C_{i, \ell+1}\left(\gamma_{i, \ell+1}\right)$ is uniformly distributed in $\mathbb{F}^{\ell+1}$, even given all objects selected before $C_{i, \ell+1}$, it holds that the objects selected after $C_{i, \ell+1}$ are independent of all objects selected before $C_{i, \ell+1}$. In particular, $Y$ is independent of the pair $\left(X, \delta_{i, \ell}\right)$. 
Proposition 4. Let $i \in I_{-d} . \delta_{i, 2 m}$ is independent of $I n f_{0}$. That is, $\delta_{i, 2 m}$ is uniformly distributed in $\mathbb{F} \backslash\{0\}$, even given $\operatorname{Inf} f_{0}$.

Proof. Clearly, $\delta_{i, 2 m}$ is uniformly distributed, even given all the previously selected objects in $\operatorname{In} f_{0}$. In addition, all the objects selected after $z_{i+1}$ are independent of all the objects selected up to $z_{i+1}$ (including $z_{i+1}$ ). Therefore, it suffices to show that $z_{i+1}$ itself is independent of all the objects selected up to $\delta_{i, 2 m}$ (including $\delta_{i, 2 m}$ ).

The curve $Z_{i+1}$ was selected to be a random degree- 2 curve satisfying $Z_{i+1}(0)=w_{i, 0}$ and $Z_{i+1}(1)=$ $w_{i, 1}$. Therefore, for every fixed $\zeta \in \mathbb{F} \backslash\{0,1\}$, the value $Z_{i+1}(\zeta)$ is uniformly distributed in $\mathbb{F}^{m}$, even given all the objects selected before $Z_{i+1}$. In particular, $z_{i+1}=Z_{i+1}\left(\zeta_{i+1}\right)$ is uniformly distributed in $\mathbb{F}^{m}$, even given all the objects selected up to $\delta_{i, 2 m}$ (including $\delta_{i, 2 m}$ ).

Let $i \in I_{-d}, \ell \in L_{-2 m}$. Proposition 5 shows that if $\mathrm{P}_{1}$ responds with the correct polynomial $T_{i, \ell+1}$, then the player can compute by herself the correct value of $Q_{i, \ell}\left(\delta_{i, \ell}\right)$.

Proposition 5. Let $i \in I_{-d}, \ell \in L_{-2 m}$. It hold that

$$
\sum_{\alpha \in \mathbb{H}} T_{i, \ell+1}^{*}\left(\zeta_{i}, \alpha\right)=Q_{i, \ell}^{*}\left(\delta_{i, \ell}\right)
$$

Proof.

$$
\begin{aligned}
\sum_{\alpha \in \mathbb{H}} T_{i, \ell+1}^{*}\left(\zeta_{i}, \alpha\right) & =\sum_{\alpha \in \mathbb{H}} \Phi_{i, \ell+1}\left(Z_{i}\left(\zeta_{i}\right), C_{i, \ell+1}(\alpha)\right) \\
& =\sum_{\alpha \in \mathbb{H}} \Phi_{i, \ell+1}\left(z_{i}, a_{i, \ell} \circ \alpha\right) \\
& =\Phi_{i, \ell}\left(z_{i}, a_{i, \ell}\right) \\
& =\Phi_{i, \ell}\left(z_{i}, D_{i, \ell}\left(\delta_{i, \ell}\right)\right) \\
& =Q_{i, \ell}^{*}\left(\delta_{i, \ell}\right)
\end{aligned}
$$

Fix strategies for $\mathrm{P}_{1}$ and $\mathrm{P}_{0}$. Formally, a strategy for $\mathrm{P}_{1}$ is a probabilistic algorithm that gets the sets of curves $Z_{i}$ and $C_{i, \ell}$ as inputs, and outputs the sets of polynomials $T_{i, \ell}$ and $U_{i}$. A strategy for $\mathrm{P}_{0}$ is a probabilistic algorithm that gets the set of points $z_{i}$ and set of curves $D_{i, \ell}$ as inputs, and outputs the set of polynomials $Q_{i, \ell}$. For the rest of the section probabilities will be taken over $\mathrm{P}_{1}$ 's random string $R_{1}, \mathrm{P}_{0}$ 's random string $R_{0}$, and the player's random string.

Provers following the reply protocol should each send a set of polynomials to the player. The player's decision protocol checks the correctness of these polynomials in a pre-determined order. Given the sets of polynomials sent by the provers, we call a pair $F=(j, G)$ a "reply polynomial" if the $j^{\text {th }}$ polynomial to be checked by the player is $G$. We will sometimes abuse notations and refer to $G$ itself as a reply polynomial (suppressing the index $j$ ).

Let $F=(j, G)$ be a reply polynomial. Denote by $E_{F}$ the event that $F$ is the first incorrect reply polynomial checked by the protocol. In other words, $E_{F}$ is the event that all the $j-1$ reply polynomials checked prior to $F$ are correct, but $F$ is incorrect (that is, had the provers followed the reply protocol, the $j^{\text {th }}$ polynomial to be checked by the player should not have been $G$ ). 


\section{Competing Provers Protocols for Circuit Evaluation}

Proposition 6. Let $F$ be a reply polynomial sent by $\mathrm{P}_{1}$. Let $X$ be a random variable that is independent of the pair $\left(\operatorname{In} f_{1}, R_{1}\right)$. Then, $X$ is independent of $\left(\operatorname{In} f_{1}, R_{1}, I_{E_{F}}\right)$, where $I_{E_{F}}$ is the random variable that gets the value 1 if $E_{F}$ occurs, and 0 otherwise.

The same holds when the parts of $\mathrm{P}_{1}$ and $\mathrm{P}_{0}$ are switched.

Proof. Assume that $E_{F}$ occurs with positive probability. $\mathrm{P}_{1}$ is dishonest as he sent an incorrect polynomial $F$ with positive probability. Since we assume that at least one prover is honest, it must be $\mathrm{P}_{0}$. Therefore, all the reply polynomials sent by $\mathrm{P}_{0}$ are correct. This implies that the event $E_{F}$ depends solely on $\operatorname{In} f_{1}$ and $R_{1}$. That is, $I_{E_{F}}$ is a function of $I n f_{1}$ and $R_{1}$. Since $X$ is independent of $\left(\operatorname{In} f_{1}, R_{1}\right)$, it is independent of $\left(\operatorname{Inf} f_{1}, R_{1}, I_{E_{F}}\right)$.

\subsection{The Player's Decision}

First, the player queries $\tilde{x}\left(z_{d}\right)$ and denote the value by $v_{d}$. Note that $\tilde{x}=\operatorname{LDE}_{s}(x)=\tilde{V}_{d}$, and thus $v_{d}=\tilde{V}_{d}\left(z_{d}\right)$. Next, the player iterates over the layers of $C$, from the layer adjacent to the input layer (layer $i=d-1$ ), to the output layer (layer $i=0$ ). The iteration that handles layer $i$ (simply referred to as "iteration $i$ ") assumes that the variable $v_{i+1}$ is set to the value $\tilde{V}_{i+1}\left(z_{i+1}\right)$. The goal of iteration $i$ is to calculate the value $v_{i}=\tilde{V}_{i}\left(z_{i}\right)$. At the end of the loop the player returns $v_{0}$, which is, supposedly, $v_{0}=\tilde{V}_{0}\left(z_{0}\right)=\tilde{V}_{0}\left(0^{m}\right)=C(x)$.

In order to compute $v_{i}$, iteration $i$ proceeds in a series of "check steps" (tests), each designed to check the correctness of one of the reply polynomials for layer $i$. Let $F$ be a reply polynomial, and let $S_{F}$ be the check step designed to check $F$. If $S_{F}$ succeeds, the protocol proceeds to the next check step. If $S_{F}$ fails, the player outputs the value of $C(x)$ claimed by the prover that did not send $F$. The reason is that we assume that at least one of the provers is honest. Since $F$ is incorrect, the prover that did not send $F$ must be the honest one.

Recall that $E_{F}$ denotes the event that $F$ is the first incorrect reply polynomial checked by the protocol. Denote by $C_{F}$ the event that all reply polynomials checked up to $F$ (including $F$ ) are correct. In order to prove Theorem 3.4 it suffices to show that for every reply polynomial $F$ the following holds:

1. If $C_{F}$ occurs, then $S_{F}$ always succeeds.

2. If $E_{F}$ occurs, then with probability at least $1-\varepsilon, S_{F}$ fails.

3. If all the reply polynomials are correct, then the player returns $C(x)$.

Next, we describe the check steps that compose iteration $i$, and show that each of them satisfies both (1) and (2). Assume for simplicity that the reply polynomial $F$ depends on only one variable. The check step $S_{F}$ does the following: It computes $F(\sigma)$, where $\sigma \in \mathbb{F}$ is a field element that cannot be predicted by the prover that sent $F$. It then compares $F(\sigma)$ against the supposed value of $F^{*}(\sigma)$ (this latter value is computed by earlier check steps). If $F$ is correct then $F=F^{*}$, and the test succeeds. If $F$ is incorrect, $F$ and $F^{*}$ are different low-degree polynomials. Thus, it is likely that $F(\sigma) \neq F^{*}(\sigma)$, and the test is likely to fail.

We assume by induction (on the layers $i$ ) that if all the reply polynomials checked by iterations $d-1, \ldots, i+1$ are correct, then at the beginning of iteration $i$ it holds that $v_{i+1}=\tilde{V}_{i+1}\left(z_{i+1}\right)$. 
Check $U_{i+1}$ : Check $U_{i+1}\left(\zeta_{i+1}\right)=v_{i+1}$. If not, return 0 .

If $C_{U_{i+1}}$ occurs: By assumption, $v_{i+1}=\tilde{V}_{i+1}\left(z_{i+1}\right)=\tilde{V}_{i+1}\left(Z_{i+1}\left(\zeta_{i+1}\right)\right)=U_{i+1}^{*}\left(\zeta_{i+1}\right)$. Since $U_{i+1}$ is correct, the test passes.

If $E_{U_{i+1}}$ occurs: Using Propositions 2 and $6, \zeta_{i+1}$ is uniformly distributed in $\mathbb{F} \backslash\{0,1\}$, even given Inf $f_{1}$, $R_{1}$ and $E_{U_{i+1}}$. Since $U_{i+1}$ only depends on $I n f_{1}$ and $R_{1}$, it holds that when $E_{U_{i+1}}$ occurs, $\zeta_{i+1}$ is uniformly distributed, even given $U_{i+1}$.

As explained above, $v_{i+1}=U_{i+1}^{*}\left(\zeta_{i+1}\right)$. Since $U_{i+1}$ is incorrect, $U_{i+1} \neq U_{i+1}^{*}$. Since both $U_{i+1}$ and $U_{i+1}^{*}$ are of degrees less than $6 m h^{2}$ (see the remark regarding functions representation at the beginning of the section), $U_{i+1}(\zeta)$ can only agree with $U_{i+1}^{*}(\zeta)$ on less than $6 m h^{2}$ elements $\zeta \in \mathbb{F}$.

Recall that when $E_{U_{i+1}}$ occurs, $\zeta_{i+1}$ is uniformly distributed in $\mathbb{F} \backslash\{0,1\}$, even given $U_{i+1}$, and we get that the probability that $U_{i+1}\left(\zeta_{i+1}\right)=U_{i+1}^{*}\left(\zeta_{i+1}\right)=v_{i+1}$ is less than $\frac{6 m h^{2}}{|\mathbb{F} \backslash\{0,1\}|}$. Since $|\mathbb{F}| \geq 12 m h^{3}$, it holds that $\frac{6 m h^{2}}{|\mathbb{F} \backslash\{0,1\}|}<\frac{6 m h^{2}}{\frac{1}{2}|\mathbb{F}|} \leq \frac{1}{h} \leq \frac{1}{\log (s)}=\varepsilon$.

Check $Q_{i, 2 m}$ : Calculate the supposed value $\phi_{i, 2 m}$ of $\Phi_{i, 2 m}\left(z_{i}, a_{i, 2 m}\right)=\Phi_{i}\left(z_{i}, w_{i, 0} \circ w_{i, 1}\right)$ (see Equation (5.2) and Lemma 5.2) using $U_{i+1}(0)$ as $\tilde{V}_{i+1}\left(w_{i, 0}\right)$ and $U_{i+1}(1)$ as $\tilde{V}_{i+1}\left(w_{i, 1}\right)$.

Check $Q_{i, 2 m}\left(\delta_{i, 2 m}\right)=\phi_{i, 2 m}$. If not, return 1.

If $C_{Q_{i, 2 m}}$ occurs: Since $U_{i+1}$ is correct $U_{i+1}(0)=U_{i+1}^{*}(0)=\tilde{V}_{i+1}\left(Z_{i+1}(0)\right)=\tilde{V}_{i+1}\left(w_{i, 0}\right)$, and similarly $U_{i+1}(1)=\tilde{V}_{i+1}\left(w_{i, 1}\right)$. Thus, $\phi_{i, 2 m}$ is set to $\Phi_{i, 2 m}\left(z_{i}, a_{i, 2 m}\right)=\Phi_{i, 2 m}\left(z_{i}, D_{i, 2 m}\left(\boldsymbol{\delta}_{i, 2 m}\right)\right)=Q_{i, 2 m}^{*}\left(\boldsymbol{\delta}_{i, 2 m}\right)$. Since $Q_{i, 2 m}$ is correct, the test passes.

If $E_{Q_{i, 2 m}}$ occurs: Using Propositions 4 and $6, \delta_{i, 2 m}$ is uniformly distributed in $\mathbb{F} \backslash\{0\}$, even given $\operatorname{In} f_{0}$, $R_{0}$ and $E_{Q_{i, 2 m}}$. Since $Q_{i, 2 m}$ only depends on $\operatorname{In} f_{0}$ and $R_{0}$, it holds that when $E_{Q_{i, 2 m}}$ occurs, $\delta_{i, 2 m}$ is uniformly distributed, even given $Q_{i, 2 m}$.

As explained above, $\phi_{i, 2 m}$ is set to $Q_{i, 2 m}^{*}\left(\delta_{i, 2 m}\right)$. Since $Q_{i, 2 m}$ is incorrect, $Q_{i, 2 m} \neq Q_{i, 2 m}^{*}$. An argument similar to the one used in check step $S_{U_{i+1}}$ shows that the probability that $Q_{i, 2 m}\left(\delta_{i, 2 m}\right)=Q_{i, 2 m}^{*}\left(\delta_{i, 2 m}\right)=$ $\phi_{i, 2 m}$ is less than $\varepsilon$.

Check $T_{i, 2 m}$ : Check $T_{i, 2 m}\left(\zeta_{i}, \gamma_{i, 2 m}\right)=Q_{i, 2 m}(0)$. If not, return 0 .

If $C_{T_{i, 2 m}}$ occurs:

$$
T_{i, 2 m}^{*}\left(\zeta_{i}, \gamma_{i, 2 m}\right)=\Phi_{i, 2 m}\left(Z_{i}\left(\zeta_{i}\right), C_{i, 2 m}\left(\gamma_{i, 2 m}\right)\right)=\Phi_{i, 2 m}\left(z_{i}, D_{i, 2 m}(0)\right)=Q_{i, 2 m}^{*}(0) .
$$

Since $Q_{i, 2 m}$ and $T_{i, 2 m}$ are correct, the test passes.

If $E_{T_{i, 2 m}}$ occurs: Using Propositions 1,2 and 6 , the pair $\left(\zeta_{i}, \gamma_{i, 2 m}\right)$ is uniformly distributed in $(\mathbb{F} \backslash\{0,1\}) \times(\mathbb{F} \backslash \mathbb{H})$, even given $\operatorname{In} f_{1}, R_{1}$ and $E_{T_{i, 2 m}}$. Since $T_{i, 2 m}$ only depends on $\operatorname{In} f_{1}$ and $R_{1}$, it holds that when $E_{T_{i, 2 m}}$ occurs, the pair $\left(\zeta_{i}, \gamma_{i, 2 m}\right)$ is uniformly distributed, even given $T_{i, 2 m}$.

Since $Q_{i, 2 m}$ is correct but $T_{i, 2 m}$ is incorrect, it holds that $Q_{i, 2 m}=Q_{i, 2 m}^{*}$ and $T_{i, 2 m} \neq T_{i, 2 m}^{*}$. Using the Schwartz Zippel Lemma (Lemma 4.1), since both $T_{i, 2 m}$ and $T_{i, 2 m}^{*}$ are of total degree less than $6 m h^{2}$ (see the remark regarding functions representation at the beginning of the section), $T_{i, 2 m}(\zeta, \gamma)$ can only agree with $T_{i, 2 m}^{*}(\zeta, \gamma)$ on less than $\frac{6 m h^{2}}{|\mathbb{F}|}$-fraction of the points $(\zeta, \gamma) \in \mathbb{F}^{2}$.

Recall that when $E_{T_{i, 2 m}}$ occurs, the pair $\left(\zeta_{i}, \gamma_{i, 2 m}\right)$ is uniformly distributed in $(\mathbb{F} \backslash\{0,1\}) \times(\mathbb{F} \backslash \mathbb{H})$, even given $T_{i, 2 m}$, and get that the probability that $T_{i, 2 m}\left(\zeta_{i}, \gamma_{i, 2 m}\right)=T_{i, 2 m}^{*}\left(\zeta_{i}, \gamma_{i, 2 m}\right)=Q_{i, 2 m}^{*}(0)=Q_{i, 2 m}(0)$ is less than $\frac{6 m h^{2} \cdot|\mathbb{F}|}{|(\mathbb{F} \backslash\{0,1\}) \times(\mathbb{F} \backslash \mathbb{H})|}$. Since $|\mathbb{F}| \geq 12 m h^{3}$, it holds that $\frac{6 m h^{2} \cdot|\mathbb{F}|}{|(\mathbb{F} \backslash\{0,1\}) \times(\mathbb{F} \backslash \mathbb{H})|}<\frac{6 m h^{2} \cdot|\mathbb{F}|}{\frac{1}{2}|\mathbb{F}|^{2}} \leq \frac{1}{h} \leq \frac{1}{\log (s)}=\varepsilon$. 
Check $Q_{i, 2 m-1}$ : Check $Q_{i, 2 m-1}\left(\delta_{i, 2 m-1}\right)=\sum_{\alpha \in \mathbb{H}} T_{i, 2 m}\left(\zeta_{i}, \alpha\right)$. If not, return 1.

If $C_{Q_{i, 2 m-1}}$ occurs: Using Proposition 5, since $T_{i, 2 m}$ and $Q_{i, 2 m-1}$ are correct, the test passes.

If $E_{Q_{i, 2 m-1}}$ occurs: Using Propositions 3 and $6, \delta_{i, 2 m-1}$ is uniformly distributed in $\mathbb{F} \backslash\{0\}$, even given $\operatorname{Inf} f_{0}, R_{0}$ and $E_{Q_{i, 2 m-1}}$. Since $Q_{i, 2 m-1}$ only depends on $I n f_{0}$ and $R_{0}$, it holds that when $E_{Q_{i, 2 m-1}}$ occurs, $\delta_{i, 2 m-1}$ is uniformly distributed, even given $Q_{i, 2 m-1}$.

Using Proposition 5, since $T_{i, 2 m}$ is correct,

$$
\sum_{\alpha \in \mathbb{H}} T_{i, 2 m}\left(\zeta_{i}, \alpha\right)=Q_{i, 2 m-1}^{*}\left(\delta_{i, 2 m-1}\right) .
$$

Since $Q_{i, 2 m-1}$ is incorrect, $Q_{i, 2 m-1} \neq Q_{i, 2 m-1}^{*}$. An argument similar to the one used in check step $S_{U_{i+1}}$ shows that the probability that $Q_{i, 2 m-1}\left(\delta_{i, 2 m-1}\right)=Q_{i, 2 m-1}^{*}\left(\delta_{i, 2 m-1}\right)=\sum_{\alpha \in \mathbb{H}} T_{i, 2 m}\left(\zeta_{i}, \alpha\right)$ is less than $\varepsilon$.

Repeat the last two steps to check $T_{i, 2 m-1}, Q_{i, 2 m-2}, \ldots, T_{i, 1}, Q_{i, 0}$

Set $v_{i}=Q_{i, 0}(\beta)$. Note that if $Q_{i, 0}$ is correct then using Equation (5.1), $v_{i}=Q_{i, 0}(\beta)=Q_{i, 0}^{*}(\beta)=$ $\Phi_{i, 0}\left(z_{i}, D_{i, 0}(\beta)\right)=\tilde{V}_{i}\left(z_{i}\right)$, and the induction hypothesis holds. In particular, if all reply polynomials are correct then $v_{0}=\tilde{V}_{0}\left(z_{0}\right)=\tilde{V}_{0}\left(0^{m}\right)=C(x)$

\section{Trade-off Protocol}

In this section we prove Theorem 3.3. We describe a protocol that given a size $s$ depth $d$ circuit $C$ on $n$ bits, and an input $x \in\{0,1\}^{n}$, outputs $C(x)$ with probability at least $1-\varepsilon$. The new protocol uses the one-round protocol described in Section 6 as a building block. The new protocol consists of $r$ rounds, requires the exchange of $d^{\frac{1}{r}} \cdot \operatorname{polylog}(s)$ bits, and makes a single query to $\operatorname{LDE}_{s}(x)$.

Let $1 \leq r \leq\lfloor\log (d)\rfloor$. Set $k=2\left\lceil d^{\frac{1}{r}}\right\rceil$. The new protocol consists of two stages. The goal of the first stage (Subsection 7.1) is to find two "close" layers $i^{\prime}, i^{\prime \prime} \in I$ with $i^{\prime}>i^{\prime \prime}$ and $i^{\prime}-i^{\prime \prime} \leq k$, such that the provers agree on a point on $\tilde{V}_{i^{\prime}}$, but disagree on a point on $\tilde{V}_{i^{\prime \prime}}$. Note that such layers exist, as the provers agree on the input $x$ for $C$ (and thus on $\operatorname{LDE}_{s}(x)=\tilde{V}_{d}$ ), but disagree on the output $C(x)$ (and therefore on $\left.\tilde{V}_{0}\right)$.

Finding layers $i^{\prime}$ and $i^{\prime \prime}$ is done by running a version of a binary search that in every round partitions the search range into $k$ (almost equal) intervals (instead of just two). Note that $r-1$ search rounds suffice in order to find such layers $i^{\prime}$ and $i^{\prime \prime}$ satisfying $i^{\prime}-i^{\prime \prime} \leq k$ : Each search round reduces the range of indices searched by a factor of at least $\frac{k}{2}$. Thus, after $r-1$ rounds we are left with a range of at most

$$
(d+1) \cdot\left(\frac{k}{2}\right)^{-(r-1)}=(d+1)\left(\left[d^{\frac{1}{r}}\right\rceil\right)^{-(r-1)} \leq 2 d\left(d^{\frac{1}{r}}\right)^{-(r-1)} \leq 2 d \cdot d^{\frac{1-r}{r}}=2 d^{\frac{1}{r}} \leq k .
$$

Let $C^{\prime}$ a subcircuit composed of layers $i^{\prime}, \ldots, i^{\prime \prime}$ of $C$, and let $x^{\prime}=V_{i^{\prime}}$. The provers agree on a point on the input layer $\operatorname{LDE}_{s}\left(x^{\prime}\right)=\tilde{V}_{i^{\prime}}$ of $C^{\prime}$, but disagree on a point on the output layer $\tilde{V}_{i^{\prime \prime}}$ of $C^{\prime}$.

Stage 2 of the new protocol (Subsection 7.2) runs the one-round protocol described in Section 6 with $C^{\prime}$ and $x^{\prime}$ as inputs in order to determine which of the provers is truthful. 


\subsection{Stage 1: Search}

First, the player constructs questions for the provers as in Subsection 6.1. That is, the player constructs the curves $C_{i, \ell}, D_{i, \ell}\left(i \in I_{-d}, \ell \in L\right)$, and the curves $Z_{i}$ and points $z_{i}(i \in I)$. For $i \in I$, let $U_{i}^{*}: \mathbb{F} \rightarrow \mathbb{F}$ be the function $U_{i}^{*}(r)=\tilde{V}_{i}\left(Z_{i}(r)\right)$, and let $u_{i}^{*} \in \mathbb{F}$ be the value $u_{i}^{*}=\tilde{V}_{i}\left(z_{i}\right)$.

Next, the protocol proceeds in $r-1$ search rounds. In each round the player constructs a set of $k$ indices $M \subseteq I$ ( $M$ contains indices that partition the remaining search range into almost equal parts). The player sends the curves $Z_{i}(i \in M)$ to $\mathrm{P}_{1}$, and the points $z_{i}(i \in M)$ to $\mathrm{P}_{0}$. $\mathrm{P}_{1}$ replies with the functions $U_{i}: \mathbb{F} \rightarrow \mathbb{F}(i \in M)$, and $\mathrm{P}_{0}$ replies with the values $u_{i} \in \mathbb{F}(i \in M)$. The honest $\mathrm{P}_{1}$ should reply with $U_{i}=U_{i}^{*}$, and the honest $\mathrm{P}_{0}$ should reply with $u_{i}=u_{i}^{*}$.

The goal of Stage 1 is to find two layers $i^{\prime}>i^{\prime \prime} \in I, i^{\prime}-i^{\prime \prime} \leq k$ such that $U_{i^{\prime}}\left(\zeta_{i^{\prime}}\right)=u_{i^{\prime}}$, but $U_{i^{\prime \prime}}\left(\zeta_{i^{\prime \prime}}\right) \neq u_{i^{\prime \prime}}$. By comparing the values of $U_{i}\left(\zeta_{i}\right)$ and $u_{i}(i \in M)$, the player decides on the smaller search range for the next round, until the desired layers $i^{\prime}$ and $i^{\prime \prime}$ are found.

Note that during the first round the player checks the provers agreement on the input and output layers. If $U_{0}\left(\zeta_{0}\right)=u_{0}$ (the provers agree on the output $C(x)$ ), then since at least one of the provers is truthful, the player outputs $u_{0}$. If $U_{d}\left(\zeta_{d}\right) \neq u_{d}$ (the provers disagree on a point in the low-degree extension $\tilde{x}=\tilde{V}_{d}$ of the input), the player queries $\tilde{x}\left(z_{d}\right)$. Since at least one of the provers is truthful, the queried value $\tilde{x}\left(z_{d}\right)$ is either $U_{d}\left(\zeta_{d}\right)$ or $u_{d}$. If $\tilde{x}\left(z_{d}\right)=U_{d}\left(\zeta_{d}\right)$, the player outputs 1 . If $\tilde{x}\left(z_{d}\right)=u_{d}$, the player outputs 0 .

\subsection{Stage 2: Run the One-Round Protocol}

Let $C^{\prime}$ be the circuit composed of layers $i^{\prime}, \ldots, i^{\prime \prime}$ of $C$. Run the one-round protocol on $C^{\prime}$ and $x^{\prime}=V_{i}$, using the questions that were already constructed in Stage 1, and with the following changes. Return the obtained value.

- Instead of querying $\operatorname{LDE}_{s}\left(x^{\prime}\right)$ at the point $z_{i^{\prime}}$, use the value $u_{i^{\prime}}$.

- Instead of returning the computed value $v_{0}$ in the last step, return 0 if $v_{0}=u_{i^{\prime \prime}}$ and 1 otherwise.

\section{Very Efficient Player}

In this section we prove Theorem 3.6. That is, we assume in addition to the previous assumptions that the circuit $C$ is $\log (s)$-space uniform and show that in this case the player can run in time $d^{\frac{1}{r}}$ polylog $(s)$.

First observe that each of the check steps of the protocol described in Section 6, excluding the check steps $S_{Q_{i, 2 m}}\left(i \in I_{-d}\right)$, can be preformed by the player in polylog $(s)$ time and without knowing the circuit $C$. The only check steps that require knowing the circuit $C$ are the check steps $S_{Q_{i, 2 m}}\left(i \in I_{-d}\right)$, as explained next.

The check step $S_{Q_{i, 2 m}}\left(i \in I_{-d}\right)$ requires the player to compute the value $\Phi_{i}\left(z_{i}, w_{i, 0}, w_{i, 1}\right)$ given the (supposed) values of $\tilde{V}_{i+1}\left(w_{i, 0}\right)$ and $\tilde{V}_{i+1}\left(w_{i, 1}\right)$. Recall that $\Phi_{i}$ was defined in Lemma 5.2 as $\Phi_{i}\left(z, w_{0}, w_{1}\right)=\tilde{\beta}_{i}\left(z, w_{0}, w_{1}\right) \tilde{V}_{i+1}\left(w_{0}\right) \tilde{V}_{i+1}\left(w_{1}\right)$. Therefore, in order to compute $\Phi_{i}\left(z_{i}, w_{i, 0}, w_{i, 1}\right)$, the player needs to know $\tilde{\beta}_{i}\left(z_{i}, w_{i, 0}, w_{i, 1}\right)$. Note that $\tilde{\beta}_{i}$ depends on the circuit $C$, and that for a general size $s$ depth $d$ circuit $C$, the evaluation of $\tilde{\beta}_{i}$ may be pricy and require poly $(s)$ time. Moreover, even in the case where the circuit $C$ is $\log (s)$-space uniform, the evaluation of $\tilde{\beta}_{i}$ by the player may require poly $(s)$ time. 


\section{Competing Provers Protocols for Circuit Evaluation}

The crucial observation is that in the case of a $\log (s)$-space uniform circuit $C$, the function $\tilde{\beta}_{i}$ is computable in space $O(\log (s))$. Note, that the inputs for the function $\tilde{\beta}_{i}$ are themselves of length $O(\log (s))$. Hence, the function $\tilde{\beta}_{i}$ can be computed in space polynomial in the length of its inputs, that is $\tilde{\beta}_{i} \in$ PSPACE.

Recall that for every language in PSPACE, Feige and Kilian give a one-round competing provers protocol that only requires polynomial communication complexity [3]. Therefore, there is a one-round

competing provers protocol that computes $\tilde{\beta}_{i}$, with communication complexity polynomial in the length of the inputs for $\tilde{\beta}_{i}$, that is, communication complexity of polylog $(s)$. Thus, the player cannot evaluate the function $\tilde{\beta}_{i}$ in time polylog $(s)$ by herself, but she can do that with the help of the two competing provers.

This naturally leads to an $r+2$ rounds protocol (rather than the $r$ rounds protocol that we want). We start by describing the $r+2$ rounds protocol and then show how to collapse the last two rounds of the protocol to round $r$ of the protocol.

Remark 8.1. We remark that when applying the competing provers protocol of Feige and Kilian for $\log (s)$-space, the provers' running time may be super-polynomial in $s$, and thus not efficient enough for our purposes. Therefore, for the rest of the paper we consider an updated version of the Feige and Kilian protocol, obtained using our protocol, as follows. A similar approach was taken in [5] (and see also [7]).

By Lemma 3.4.3 of [10], any $\log (s)$-space language has a poly $(s)$-size polylog $(s)$-depth arithmetic circuit $C$, for which the associated functions $\tilde{\beta}_{i}$ have polylog $(s)$-size arithmetic circuits. Therefore, the following is a competing provers protocol for $\log (s)$-space, with efficient parties: Given a $\log (s)$-space language, the parties run our 1-round competing provers protocol with the circuit $C$. Observe that since $C$ has poly $(s)$-size and polylog $(s)$-depth, the provers run in time poly $(s)$. In addition, since the associated functions $\tilde{\beta}_{i}$ have polylog $(s)$-size circuits, the player can evaluate $\tilde{\beta}_{i}$ by herself in polylog $(s)$ time.

\subsection{The $r+2$ Rounds Protocol}

The first $r$ rounds of the protocol run the $r$ rounds of the protocol of Section 7, except for the player's decision that is still not made. In order to make her decision, the player needs to evaluate $\tilde{\beta}_{i}\left(z_{i}, w_{i, 0}, w_{i, 1}\right)$ for each of the at most $k$ layers $i \in\left\{i^{\prime \prime}, \ldots, i^{\prime}-1\right\}$ that round $r$ of the protocol considers (see Subsection 7.2).

In order to compute the needed values of $\tilde{\beta}_{i}\left(z_{i}, w_{i, 0}, w_{i, 1}\right)$, the player sends in round $r+1$ the values $\left(z_{i}, w_{i, 0}, w_{i, 1}\right)$ (for $i \in\left\{i^{\prime \prime}, \ldots, i^{\prime}-1\right\}$ ) to both provers, and asks each prover to reply with the values of $\tilde{\beta}_{i}\left(z_{i}, w_{i, 0}, w_{i, 1}\right)$ (for $i \in\left\{i^{\prime \prime}, \ldots, i^{\prime}-1\right\}$ ). If the provers agree on all the values $\tilde{\beta}_{i}\left(z_{i}, w_{i, 0}, w_{i, 1}\right)$, the player knows that these values are correct (because at least one of the provers is honest), and can proceed with her decision. If the provers disagree on one or more of the values $\tilde{\beta}_{i}\left(z_{i}, w_{i, 0}, w_{i, 1}\right)$, the player picks one such value and applies in round $r+2$ the PSPACE protocol of Feige and Kilian in order to identify the correct value and decide which prover is honest.

Next, we show how to reduce the number of rounds from $r+2$ to $r$.

\subsection{Collapsing Round $r+1$ to Round $r$}

We now describe how to collapse round $r+1$ of the protocol to round $r$. We cannot just send all the values $\left(z_{i}, w_{i, 0}, w_{i, 1}\right)$ (for $i \in\left\{i^{\prime \prime}, \ldots, i^{\prime}-1\right\}$ ) to both provers at the beginning of round $r$, because this would give each prover additional information that may enable him to cheat in round $r$. 
Note, however, that at the beginning of round $r$, the prover $\mathrm{P}_{1}$ gets all the curves $Z_{i}$ (for $i \in\left\{i^{\prime \prime}, \ldots, i^{\prime}\right\}$ ). Hence, for $i \in\left\{i^{\prime \prime}, \ldots, i^{\prime}-1\right\}$, the prover $\mathrm{P}_{1}$ already knows the needed values of $w_{i, 0}, w_{i, 1}$ (since $Z_{i+1}(0)=$ $w_{i, 0}$ and $\left.Z_{i+1}(1)=w_{i, 1}\right)$, as well as the curve $Z_{i}$ that contains the point $z_{i}$. Thus, we can just ask $\mathrm{P}_{1}$ for the values of $\tilde{\beta}_{i}\left(z_{i}^{\prime}, w_{i, 0}, w_{i, 1}\right)$ for all the points $z_{i}^{\prime}$ on the image of $Z_{i}$.

In addition, at the beginning of round $r$, the prover $\mathrm{P}_{0}$ gets all the points $z_{i}$ as well as the curves $D_{i, 2 m}$ (for $i \in\left\{i^{\prime \prime}, \ldots, i^{\prime}-1\right\}$ ). Hence, for $i \in\left\{i^{\prime \prime}, \ldots, i^{\prime}-1\right\}$, the prover $\mathrm{P}_{0}$ already knows the needed value of $z_{i}$, as well as the curve $D_{i, 2 m}$ that contains $w_{i, 0} \circ w_{i, 1}$ (since $\left.D_{i, 2 m}\left(\delta_{i, 2 m}\right)=a_{i, 2 m}=w_{i, 0} \circ w_{i, 1}\right)$. Thus, we can just ask $\mathrm{P}_{0}$ for the values of $\tilde{\beta}_{i}\left(z_{i}, w_{i, 0}^{\prime} \circ w_{i, 1}^{\prime}\right)=\tilde{\beta}_{i}\left(z_{i}, w_{i, 0}^{\prime}, w_{i, 1}^{\prime}\right)$ for all the points $w_{i, 0}^{\prime} \circ w_{i, 1}^{\prime}$ on the image of $D_{i, 2 m}$.

Denote by $A_{i}^{*}, B_{i}^{*}: \mathbb{F} \rightarrow \mathbb{F}$ the following restrictions of the function $\tilde{\beta}_{i}$ to curves: $A_{i}^{*}(t)=\tilde{\beta}_{i}\left(Z_{i}(t), w_{i, 0}, w_{i, 1}\right)$ and $B_{i}^{*}(t)=\tilde{\beta}_{i}\left(z_{i}, D_{i, 2 m}(t)\right)$ (for $i \in\left\{i^{\prime \prime}, \ldots, i^{\prime}-1\right\}$ ). The new protocol asks $\mathrm{P}_{1}$ to add to his reply message in round $r$ the polynomials $A_{i}^{*}$, and asks $\mathrm{P}_{0}$ to add to his reply message in round $r$ the polynomials $B_{i}^{*}$ (for $\left.i \in\left\{i^{\prime \prime}, \ldots, i^{\prime}-1\right\}\right)$.

Suppose that $\mathrm{P}_{1}$ replied with $A_{i}: \mathbb{F} \rightarrow \mathbb{F}$ and that $\mathrm{P}_{0}$ replied with $B_{i}: \mathbb{F} \rightarrow \mathbb{F}$ (for $i \in\left\{i^{\prime \prime}, \ldots, i^{\prime}-1\right\}$ ). Observe that $A_{i}^{*}\left(\zeta_{i}\right)=\tilde{\beta}_{i}\left(Z_{i}\left(\zeta_{i}\right), w_{i, 0}, w_{i, 1}\right)=\tilde{\beta}_{i}\left(z_{i}, w_{i, 0}, w_{i, 1}\right)$ and that $B_{i}^{*}\left(\delta_{i, 2 m}\right)=\tilde{\beta}_{i}\left(z_{i}, D_{i, 2 m}\left(\delta_{i, 2 m}\right)\right)=$ $\tilde{\beta}_{i}\left(z_{i}, a_{i, 2 m}\right)=\tilde{\beta}_{i}\left(z_{i}, w_{i, 0}, w_{i, 1}\right)$. If for every $i \in\left\{i^{\prime \prime}, \ldots, i^{\prime}-1\right\}$ it holds that $A_{i}\left(\zeta_{i}\right)=B_{i}\left(\delta_{i, 2 m}\right)$, the player may use the value $A_{i}\left(\zeta_{i}\right)=B_{i}\left(\delta_{i, 2 m}\right)$ as $\tilde{\beta}_{i}\left(z_{i}, w_{i, 0}, w_{i, 1}\right)$ and complete her decision protocol. Otherwise, she proceeds to round $r+2$.

Thus, we showed how to collapse round $r+1$ of the protocol to round $r$.

\subsection{Collapsing Round $r+2$ to Round $r$}

We now show how to collapse round $r+2$ to round $r$, resulting in an $r$ rounds protocol. Round $r+2$ is only reached if there exists $j \in\left\{i^{\prime \prime}, \ldots, i^{\prime}-1\right\}$ such that $A_{j}\left(\zeta_{j}\right) \neq B_{j}\left(\delta_{j, 2 m}\right)$. In this case, in order to decide which of the provers is truthful, the player runs the one-round protocol of [3].

In round $r+2$, if reached, the player and provers run the one-round protocol of [3] in order to determine the value of $\tilde{\beta}_{j}\left(z_{j}, w_{j, 0}, w_{j, 1}\right)$, for some $j \in\left\{i^{\prime \prime}, \ldots, i^{\prime}-1\right\}$ and some $z_{j}, w_{j, 0}, w_{j, 1}$, where $\mathrm{P}_{0}$ claims that the value is $B=B_{j}\left(\delta_{j, 2 m}\right)$ and $\mathrm{P}_{1}$ claims that the value is $A=A_{j}\left(\zeta_{j}\right)$. We would like to collapse round $r+2$ to round $r$, but the problem is that after the questions part of round $r$, the values of $j, z_{j}, w_{j, 0}, w_{j, 1}, A, B$ are still not all known to the player or the provers. Note, however, that the number of possibilities for these values is small from the point of view of each of the parties.

First note that $j$ can take at most $k$ values and $A, B$ can each take at most $p=|\mathbb{F}|$ values. Also, after the questions part of round $r$, the player and $\mathrm{P}_{1}$ already know $w_{j, 0}, w_{j, 1}$, and from the point of view of $\mathrm{P}_{0}$ the element $w_{j, 0} \circ w_{j, 1}$ can take at most $p$ values (as $w_{j, 0} \circ w_{j, 1}$ is on the image of $D_{j, 2 m}$, which is of size at most $p$ ). As for $z_{j}$, after the questions part of round $r$, the player and $\mathrm{P}_{0}$ already know $z_{j}$, and from the point of view of $\mathrm{P}_{1}$ the point $z_{j}$ can take at most $p$ values (as $z_{j}$ is on the image of $Z_{j}$, which is of size at most $p$ ).

Let $\Lambda$ be the set of all $\lambda=\left(j^{\prime}, z_{j^{\prime}}^{\prime}, w_{j^{\prime}, 0}^{\prime}, w_{j^{\prime}, 1}^{\prime}, A^{\prime}, B^{\prime}\right)$, such that, $j^{\prime} \in\left\{i^{\prime \prime}, \ldots, i^{\prime}-1\right\}$, and $z_{j^{\prime}}^{\prime}$ is on the image of $Z_{j^{\prime}}$, and $w_{j^{\prime}, 0}^{\prime} \circ w_{j^{\prime}, 1}^{\prime}$ is on the image of $D_{j^{\prime}, 2 m}$, and $A^{\prime}, B^{\prime} \in \mathbb{F}$. For every $\lambda \in \Lambda$, let $\mathcal{Q}(\boldsymbol{\lambda})=\left(Q_{0}(\lambda), Q_{1}(\lambda)\right)$ be independently chosen pair of questions for the Feige-Kilian protocol corresponding to $\lambda$ (that is, for $\lambda=\left(j^{\prime}, z_{j^{\prime}}^{\prime}, w_{j^{\prime}, 0}^{\prime}, w_{j^{\prime}, 1}^{\prime}, A^{\prime}, B^{\prime}\right)$, the Feige-Kilian protocol for evaluating 


\section{Competing Provers Protocols for Circuit Evaluation}

$\tilde{\beta_{j^{\prime}}}\left(z_{j^{\prime}}^{\prime}, w_{j^{\prime}, 0}^{\prime}, w_{j^{\prime}, 1}^{\prime}\right)$ when $\mathrm{P}_{0}$ claims that the value is $B^{\prime}$ and $\mathrm{P}_{1}$ claims that the value is $\left.A^{\prime}\right)$.

We can now collapse round $r+2$ to round $r$ as follows. In the questions part of round $r$, in addition to the questions of the original protocol of Section 7 , the player will send to $\mathrm{P}_{0}$ the pair $\left(\lambda, Q_{0}(\lambda)\right)$, for every $\lambda=\left(j^{\prime}, z_{j^{\prime}}^{\prime}, w_{j^{\prime}, 0}^{\prime}, w_{j^{\prime}, 1}^{\prime}, A^{\prime}, B^{\prime}\right) \in \Lambda$, such that, $z_{j^{\prime}}^{\prime}=z_{j^{\prime}}$. In the same way, the player will send to $\mathrm{P}_{1}$ the pair $\left(\lambda, Q_{1}(\lambda)\right)$, for every $\lambda=\left(j^{\prime}, z_{j^{\prime}}^{\prime}, w_{j^{\prime}, 0}^{\prime}, w_{j^{\prime}, 1}^{\prime}, A^{\prime}, B^{\prime}\right) \in \Lambda$, such that, $\left(w_{j^{\prime}, 0}^{\prime}, w_{j^{\prime}, 1}^{\prime}\right)=\left(w_{j^{\prime}, 0}, w_{j^{\prime}, 1}\right)$. Note that this doesn't give the provers additional information about the questions of the original protocol of Section 7, because each prover could have constructed by himself questions that are distributed the same as the additional questions that he got.

In the reply part of round $r$, the prover $\mathrm{P}_{0}$ will send his reply for the question $Q_{0}(\lambda)$ for the FeigeKilian protocol corresponding to $\lambda$, for every pair $\left(\lambda, Q_{0}(\lambda)\right)$ that he got. This is in addition to the replies for the questions of the original protocol of Section 7, and in addition to the functions $B_{i}$ (required in order to collapse round $r+1$ to round $r$, as discussed above). In the same way, the prover $\mathrm{P}_{1}$ will send his reply for the question $Q_{1}(\lambda)$ for the Feige-Kilian protocol corresponding to $\lambda$, for every pair $\left(\lambda, Q_{1}(\lambda)\right)$ that he got (this is in addition to the replies for the questions of the original protocol of Section 7 , and in addition to the functions $A_{i}$ ). Denote the reply of $\mathrm{P}_{0}$ for the question $\mathcal{Q}_{0}(\lambda)$ by $\mathcal{R}_{0}(\lambda)$, and denote the reply of $\mathrm{P}_{1}$ for the question $Q_{1}(\lambda)$ by $\mathcal{R}_{1}(\lambda)$.

The decision made by the player is as follows. If for every $i \in\left\{i^{\prime \prime}, \ldots, i^{\prime}-1\right\}$ it holds that $A_{i}\left(\zeta_{i}\right)=$ $B_{i}\left(\delta_{i, 2 m}\right)$, the player may use the value $A_{i}\left(\zeta_{i}\right)=B_{i}\left(\delta_{i, 2 m}\right)$ as $\tilde{\beta}_{i}\left(z_{i}, w_{i, 0}, w_{i, 1}\right)$ and make her decision according to the decision part of the original protocol of Section 7. If for some $j \in\left\{i^{\prime \prime}, \ldots, i^{\prime}-1\right\}$ it holds that $A_{j}\left(\zeta_{j}\right) \neq B_{j}\left(\delta_{j, 2 m}\right)$, the player decides according to the decision part of the Feige-Kilian protocol corresponding to $\lambda=\left(j, z_{j}, w_{j, 0}, w_{j, 1}, A_{j}\left(\zeta_{j}\right), B_{j}\left(\delta_{j, 2 m}\right)\right)$. This can be done since both provers have sent replies $\mathcal{R}_{0}(\lambda)$ and $\mathcal{R}_{1}(\lambda)$, respectively.

Thus, we obtained an $r$ rounds protocol. Note that the size of $\Lambda$ is $k \cdot \operatorname{polylog}(s)$. Hence, the total communication of the new protocol is $d^{\frac{1}{r}}$ polylog $(s)$, as required. Since the error of the original protocol of Section 7 is $\varepsilon$, and the error of the protocol of Feige and Kilian is negligible and in particular is smaller than $\frac{\varepsilon}{|\Lambda|}$, the total error of the new protocol is at most $2 \varepsilon$ (by the union bound).

\subsection{Running Time of the Player}

The running time of the player in the protocol described in the previous subsections is $d \cdot \operatorname{polylog}(s)$, rather than the desired $d^{\frac{1}{r}}$ polylog $(s)$. This is because in the original protocol of Section 7 , the player generates for every layer $i \in I_{-d}$ the corresponding curves and points, at the beginning of the protocol, which takes time $d \cdot \operatorname{polylog}(s)$. To reduce the running time of the player to $d^{\frac{1}{r}}$ polylog $(s)$, note that the player only needs to generate the curves and points corresponding to layers $i \in I_{-d}$ that are actually used.

More precisely, define the questions corresponding to layer $i \in I_{-d}$ to be the set of curves $C_{i, \ell}$ and $D_{i, \ell}(\ell \in L)$, the curve $Z_{i+1}$, and the point $z_{i+1}$. Note that the questions for layer $i$ can be generated independently of the questions for all the other layers.

We change the protocol, so that the player generates the questions corresponding to layer $i$ only if and when layer $i$ is used. That is, the questions corresponding to layer $i$ are generated during round $r^{\prime} \in[r]$ iff $i$ is one of the $k$ indices queried in round $r^{\prime}$. Note that since only a total number of $O(r \cdot k) \leq$ $O\left(\log (d) \cdot d^{\frac{1}{r}}\right)$ indices are queried, the player only spends $d^{\frac{1}{r}}$ polylog $(s)$ time constructing questions. 


\section{References}

[1] Scott Aaronson And Avi Wigderson: Algebrization: A new barrier in complexity theory. TOCT, 1(1), 2009. 2, 3, 4, 7

[2] Ran Canetti, Ben Riva, and Guy N. Rothblum: Two protocols for delegation of computation. In ICITS, pp. 37-61, 2012. 1, 3, 9

[3] Uriel Feige and Joe Kilian: Making games short (extended abstract). In STOC, pp. 506-516, 1997. 1, 2, 3, 4, 21, 22

[4] Uriel Feige, Adi Shamir, And Moshe Tennenholtz: The noisy oracle problem. In CRYPTO, pp. 284-296, 1988. 2

[5] Shafi Goldwasser, Yael Tauman Kalai, and Guy N. Rothblum: Delegating computation: interactive proofs for muggles. In STOC, pp. 113-122, 2008. 1, 2, 3, 4, 8, 10, 21

[6] Yael Tauman Kalai and Ran Raz: Interactive PCP. In ICALP (2), pp. 536-547, 2008. 2

[7] Yael Tauman Kalai, Ran Raz, and Ron Rothblum: How to delegate computations: The power of no-signaling proofs. In STOC, 2014. 21

[8] Gillat Kol and Ran Raz: Competing provers protocols for circuit evaluation. In ITCS, pp. 473-484, 2013. 1

[9] Daphne Koller And Nimrod Megiddo: The complexity of two-person zero-sum games in extensive form. Games and Economic Behavior, 4:528-552, 1992. 2

[10] Guy N. Rothblum: Delegating computation reliably: Paradigms and constructions. In Ph.D. Thesis - Massachusetts Institute of Technology, Dept. of Electrical Engineering and Computer Science, 2009. 21

\section{AUTHORS}

Gillat Kol

Institute for Advanced Study (IAS), Princeton, NJ

gillat.kol@gmail.com

Ran Raz

Weizmann Institute of Science, Israel

ran.raz.mail@gmail.com

http://www.wisdom.weizmann.ac.il/ /ranraz/ 


\section{ABOUT THE AUTHORS}

GILlAT KOL is a postdoctoral researcher at the school of Mathematics at the Institute for Advanced Study (IAS), Princeton. She received her Ph.D. from the Weizmann Institute, Israel. Her main research area is complexity theory, with focus on Information Theory, Interactive Communication, and analysis of Boolean functions.

RAN RAZ received his Ph.D. in 1992 from Hebrew University under the supervision of Michael Ben-Or and Avi Wigderson. Since 1994, he has been a faculty member in the Faculty of Mathematics and Computer Science at the Weizmann Institute. His main research area is complexity theory, with emphasis on proving lower bounds for computational models. More specifically, he is interested in Boolean circuit complexity, arithmetic circuit complexity, communication complexity, propositional proof theory, probabilistically checkable proofs, quantum computation and communication, and randomness and derandomization. 